\title{
Direct Probe of Dark Energy through Gravitational Lensing Effect
}

\author{
Hong-Jian He, ${ }^{a, b}$ Zhen Zhang ${ }^{b}$ \\ ${ }^{a}$ Institute of Modern Physics and Center for High Energy Physics, \\ Tsinghua University, Beijing 100084, China. \\ ${ }^{b}$ Center for High Energy Physics, Peking University, Beijing 100871, China. \\ E-mail: hjhe@tsinghua.edu.cn, zh.zhang@pku.edu.cn
}

\begin{abstract}
.
We show that gravitational lensing can provide a direct method to probe the nature of dark energy at astrophysical scales. For lensing system as an isolated astrophysical object, we derive the dark energy contribution to gravitational potential as a repulsive power-law term, containing a generic equation of state parameter $w$. We find that it generates $w$ dependent and position-dependent modification to the conventional light orbital equation of $w=-1$. With post-Newtonian approximation, we compute its direct effect for an isolated lensing system at astrophysical scales and find that the dark energy force can deflect the path of incident light rays. We demonstrate that the dark-energy-induced deflection angle $\Delta \alpha_{\mathrm{DE}} \propto M^{\left(1+\frac{1}{3 w}\right)}$ (with $1+\frac{1}{3 w}>0$ ), which increases with the lensing mass $M$ and consistently approaches zero in the limit $M \rightarrow 0$. This effect is distinctive because dark energy tends to diffuse the rays and generates concave lensing effect. This is in contrast to the conventional convex lensing effect caused by both visible and dark matter. Measuring such concave lensing effect can directly probe the existence and nature of dark energy. We estimate this effect and show that the current gravitational lensing experiments are sensitive to the direct probe of dark energy at astrophysical scales. For the special case $w=-1$, our independent study favors the previous works that the cosmological constant can affect light bending, but our predictions qualitatively and quantitatively differ from the literature, including our consistent realization of $\Delta \alpha_{\mathrm{DE}} \rightarrow 0$ (under $M \rightarrow 0$ ) at the leading order.
\end{abstract}

\section{Keywords:}

Dark Energy Theory, Gravitational Lensing, Particle Physics-Astrophysics Connection

JCAP (2017) [arXiv:1701.03418 [astro-ph.CO]]. 


\section{Contents}

1 Introduction $\quad 2$

2 Gravitational Potential Including Dark Energy 4

3 Direct Dark Energy Effect in Gravitational Lensing 5

4 Probing Concave Lensing of Dark Energy 10

5 Light Orbital Equation with Generic State Parameter w 15

6 Conclusions 16

A Dark Energy Potential and Dark Energy Models $\quad 17$

A.1 General Gravitational Potential Including Dark Energy 18

A.2 Phantom Model 21

A.3 Quintessence Model 21

A.4 Cosmological Constant Model 22

B Deriving Light Orbital Equation for Generic State Parameter w 26

C Analyses within and outside the Lensing System 27

C.1 Cosmic Expansion Factor is Nearly Constant at Astrophysical Scales 27

C.2 Approximate Conformal Flatness for Generic State Parameter w 28

$\begin{array}{lr}\text { References } & 29\end{array}$

\section{Introduction}

The discovery of cosmic acceleration has pointed to the mysterious dark energy, which composes about $69 \%$ of the total energy density of the present universe [1] and remains one of the greatest puzzles of modern science. To unravel the mystery of dark energy faces two challenges: one is to find out direct observational evidence of dark energy [2], and another is to identify the right theory for describing the dark energy [3].

The current dark energy detections [2] are mostly indirect, taking the form of large astronomical surveys and including such as the CMB measurements of WMAP and Planck, the Type-Ia Supernovae, the Baryon Acoustic Oscillations, the Gravitational Lensing, the Clusters of Galaxies, and so on. One of these approaches is the gravitational lensing [4], which has become an important method to probe both the dark matter and dark energy $[5,6]$ since its observation in 1979 [7]. So far the gravitational lensing analyses are through the indirect probe of dark energy, where the dark energy effect is indirectly included via the scale factor $a(t)$ defined under Friedmann-Lemaitre-Robertson-Walker (FLRW) metric and the matter contribution is treated as perturbation. Because the CMB measurements [1] at 
cosmological scale show that the FLRW spacetime is nearly flat (with spatial curvature $K \simeq 0$ ) and the matter perturbation only generates Newtonian deflection, the conventional lensing analyses find no measurable direct effect of dark energy, as is obvious. But, for most of the gravitational lensings at the (shorter) astrophysical scales (such as the scales of galaxies and galaxy clusters), the cosmological expansion factor $a(t)$ is nearly constant, and the dark energy effect should be best included via its direct contribution to the gravitational potential as a repulsive force in parallel to the matter contribution. This can be derived under the Schwarzschild-de Sitter spacetime with a generic equation of state parameter $w$ (denoted as $\mathrm{SdS} w$ metric), and we solve Einstein equation for the isolated lensing system in the $\mathrm{SdS} w$ spacetime (Appendix A.1).

In this work, we study the direct probe of dark energy through the gravitational lensing at astrophysical scales. We will establish a setup to define the astrophysical-scale gravitational lens as an isolated system with an effective radius $r_{\text {eff }}$ (characetrized by its critical radius $r_{\text {cri }}$ at which the dark energy repulsive force just cancels the attractive Newtonian force of matter). For such a lensing system, we use the post-Newtonian approximation to derive the general gravitational potential including both the Newtonian term and the dark energy term (with a generic equation of state parameter $w$ ) in the $\operatorname{SdS} w$ spacetime. Inside this system, we demonstrate that the dark energy force directly contributes to the lensing effect together with the Newtonian force. For the case of a single lensing, in the regions outside this isolated lensing system $\left(r^{3} \gg r_{\text {eff }}^{3} \sim r_{\text {cri }}^{3}\right)$, the Newtonian potential becomes negligible and the spacetime conformally recovers the FLRW metric with vanishing spatial curvature $K=0$, which is conformally flat and thus will not change the deflection angle of the light ray.

We will further demonstrate that the dark-energy-induced deflection angle $\Delta \alpha_{\mathrm{DE}} \propto$ $M^{\left(1+\frac{1}{3 w}\right)}$ (with $1+\frac{1}{3 w}>0$ ), which increases with the lensing mass $M$ and consistently approaches zero under the limit $M \rightarrow 0$. This effect is distinctive because dark energy tends to diffuse the rays and generates concave lensing effect, contrary to the conventional convex lensing effect caused by both visible and dark matter. Measuring such concave lensing effect can directly probe the existence and nature of dark energy. We will estimate this effect and show that the current gravitational lensing experiments are sensitive to the direct probe of dark energy at astrophysical scales. We also note that for the special case $w=$ -1 , our independent study favors the previous works of Ishak and collaborators [8][9] that the cosmological constant can affect light bending. But our predictions qualitatively and quantitatively differ from the literature [8], including our consistent realization of $\Delta \alpha_{\mathrm{DE}} \rightarrow 0$ (under $M \rightarrow 0$ ) at the leading order.

This work is organized as follows. In section 2, we introduce the dark energy potential with a generic equation of state parameter $w$, for an isolated astrophysical system. In section 3 , we analyze the dark energy lensing effect, and demonstrate that it acts as a concave 
lensing, in contrast with the conventional convex lensing effect caused by both visible and dark matter. In section 4, we estimate the dark energy lensing effect, and show that the current gravitational lensing experiments are sensitive to the direct probe of dark energy. In section 5 , for the lensing system with a point-like spherical mass-distribution, we derive a new orbital equation of light rays for a generic state parameter $w$. It generates $w$-dependent and position-dependent modification to the conventional light orbital equation of $w=-1$. We will conclude in section 6. In Appendix A, we derive the general dark energy potential used in the main text and discuss its connection with the typical dark energy models. We also clarify the difference of our independent approach from the previous studies of the cosmological constant case $(w=-1)$ [8] at the end of Appendix A.4. In Appendix B, we derive the light orbital equation in the $\mathrm{SdS} w$ spacetime. Finally, Appendix C presents two related analyses within and outside the lensing system.

\section{Gravitational Potential Including Dark Energy}

In the conventional analysis of gravitational lensing, the gravitational potential is entirely determined by matter (including visible matter and dark matter). But we note that dark energy can directly modify the form of gravitational potential at astrophysical scales. This gives rise to a correction term $\Delta \Phi$ in the gravitational potential, and should be included to directly describe the gravitational lensing effects. Following Ref. [10], we can write the metric of an isolated astrophysical system, under the post-Newtonian approximation,

$$
\mathrm{d} S^{2}=(1+2 \Phi) \mathrm{d} t^{2}-(1-2 \Phi)\left(\mathrm{d} x^{2}+\mathrm{d} y^{2}+\mathrm{d} z^{2}\right),
$$

where $\Phi=\Phi_{\mathrm{N}}+\Delta \Phi$ is the gravitational potential, containing the conventional Newtonian potential $\Phi_{\mathrm{N}}$ and the correction term $\Delta \Phi=\Phi_{\mathrm{DE}}$ as induced by dark energy. For the postNewtonian region within the effective radius $\left(r<r_{\text {eff }} \sim r_{\text {cri }}\right)$, the metric (2.1) holds well and the dark energy contributaion $\Phi_{\mathrm{DE}}$ behaves as an effective potential (cf. Appendix A.1). As we show in Eqs.(A.17) and (A.19) of Appendix A.1, for such an isolated astrophysical system, the dark energy contribution $\Phi_{\mathrm{DE}}$ to the gravitational potential $\Phi$ is unavoidable, and must be included together with the matter contribution $\Phi_{\mathrm{N}}$. Hence, we can study the direct contribution of $\Phi_{\mathrm{DE}}$ to the gravitational lensing at astrophysical scales.

In the spherical coordinate system, we locate the center of mass $M$ at the origin and adopt the geometrized unit system $(G=h=c=1)$. Then, we can solve Einstein equation exactly in Appendix A.1 and obtain the complete potential form,

$$
\Phi=\Phi_{\mathrm{N}}+\Phi_{\mathrm{DE}}=-\frac{M}{r}-\left(\frac{r_{o}}{r}\right)^{3 w+1}
$$

where the Newtonian term $\Phi_{\mathrm{N}}=-M / r$ holds for a point-like mass $M$ or for regions outside a spherically symmetric mass-distribution. For a general case of a mass-density $\rho(\mathbf{r})$ distributed over a space region $\Omega$, the total mass is $M=\int_{\Omega} \mathrm{d}^{3} \mathbf{r}^{\prime} \rho\left(\mathbf{r}^{\prime}\right)$, and the Newtonian potential is 
$\Phi_{\mathrm{N}}=-\int_{\Omega} \mathrm{d}^{3} \mathbf{r}^{\prime} \rho\left(\mathbf{r}^{\prime}\right) /\left|\mathbf{r}-\mathbf{r}^{\prime}\right|$. In Eq.(2.2), the constant $w$ is the equation of state parameter of dark energy and is given by $w=p / \rho$ as the ratio between the pressure $p$ and energy density $\rho$. The parameter $r_{o}$ of $\Phi_{\mathrm{DE}}$ characterizes the size of the present universe and will be given in each dark energy model when comparing with the cosmological data.

Eq.(2.2) shows that the dark energy induced correction term $\Phi_{\mathrm{DE}}$ takes a modelindependent form. It can describe the equation of state for different dark energy models [11]: $w=-1$ for the cosmological constant dominated state, $-1<w<-\frac{1}{3}$ for the quitessence dominated state, and $w<-1$ for the phantom dominated state. For the cosmological constant model of dark energy, the dark energy induced gravitational potential takes the form, $\Phi_{\mathrm{DE}}=-\left(r / r_{o}\right)^{2}$ with $r_{o}=\sqrt{6 / \Lambda}$, which will be discussed in Appendix-A.4.

\section{Direct Dark Energy Effect in Gravitational Lensing}

After including the direct correction of dark energy to the Newtonian potential, the metric takes the form of Eq.(2.1) in the post-Newtonian region, where the full gravitational potential $\Phi=\Phi_{\mathrm{N}}+\Phi_{\mathrm{DE}}$ contains both the matter and dark energy contributions as given by Eq.(2.2). We consider the potential to be fairly weak, $\Phi_{\mathrm{N}} \ll 1$ and $\Phi_{\mathrm{DE}} \ll 1$. Under this weak field approximation, the full potential (2.2) is derived from the Einstein equation as in Appendix A.1.

Photons propagate along the light-like path with $\mathrm{d} S^{2}=0$. Let $\mathrm{d} \ell=(\mathrm{d} x, \mathrm{~d} y, \mathrm{~d} z)$ denote the change of spatial coordinates $(x, y, z)$ of the photon during a time interval $\mathrm{d} t$. Thus, under linear approximation, we deduce from Eq. (2.1),

$$
\mathrm{d} t=\left(1-2 \Phi_{\mathrm{N}}-2 \Phi_{\mathrm{DE}}\right) \mathrm{d} \ell .
$$

In the space with gravitational field, a photon propagates precisely as if there is no gravitational field, but the space is filled with refractive medium. From Eq.(3.1), we deduce the refractive index $n=n(x, y, z)$,

$$
n=n_{\mathrm{M}}+\Delta n_{\mathrm{DE}}=1-2 \Phi_{\mathrm{N}}-2 \Phi_{\mathrm{DE}}
$$

where $n_{\mathrm{M}}$ is the conventional matter contribution, and $\Delta n_{\mathrm{DE}}$ is the dark energy induced correction term,

$$
n_{\mathrm{M}}=1-2 \Phi_{\mathrm{N}}, \quad \Delta n_{\mathrm{DE}}=-2 \Phi_{\mathrm{DE}}
$$

In Fig. 1, the red curve shows that the trajectory of a ray ${ }^{1}$ from source to observer is bent. In the post-Newtonian region, we locate the source at point $\mathcal{S}$ and the observer at point $\mathcal{O}$, while the center of mass $M$ sits at the origin $C$. The ray propagates through the

\footnotetext{
${ }^{1}$ Here, a ray generically refers to any possible ray of electromagnetic wave, such as light ray, $X$ ray, and $\gamma$ ray. (It could also be certain relativistic particles.) In the present work, we will often mention the light ray as a familiar example without losing generality.
} 


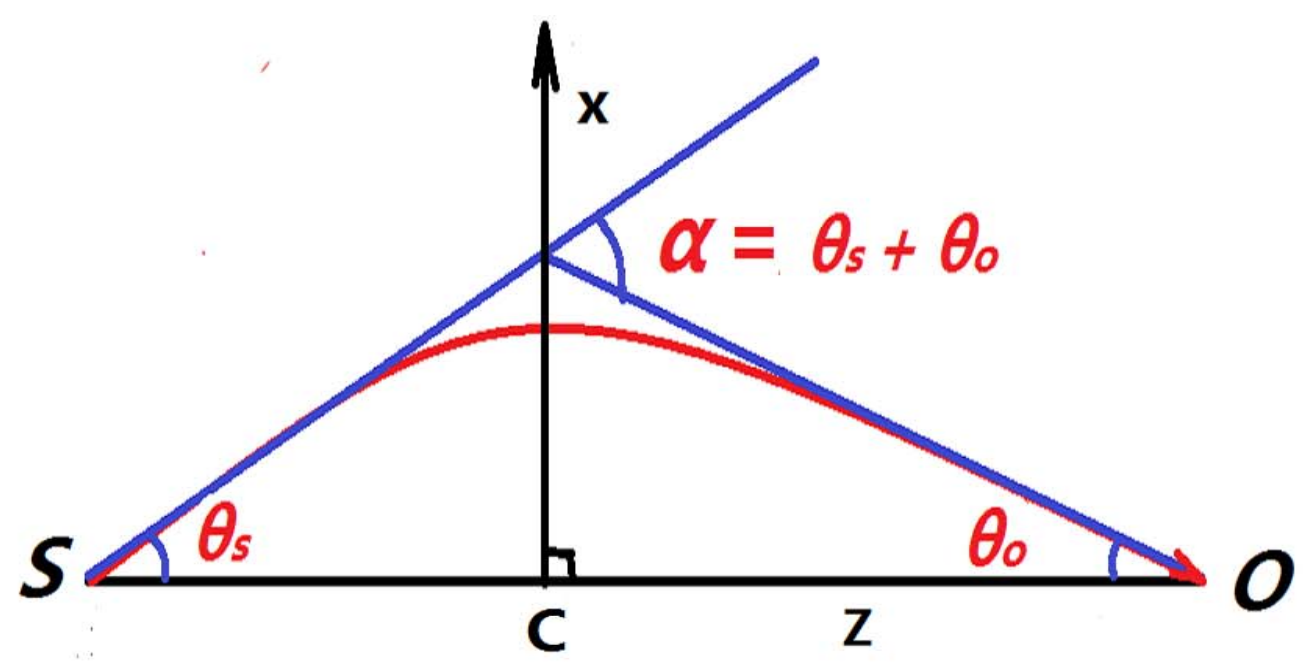

Figure 1. Convex gravitational lensing. The red curve represents the projected trajectory of a ray in the $x-z$ plane, which is deflected by the gravitational field of matter. The $\alpha$ denotes the angle between the directions of the ray at the point $\mathcal{S}$ and at the point $\mathcal{O}$. The incident angle at $\mathcal{S}$ is $\theta_{\mathcal{S}}$, and the outgoing angle at $\mathcal{O}$ is $\theta_{\mathcal{O}}$. The center of mass $M$ sits at the point $C$.

deflecting region between the two points $\mathcal{S}$ and $\mathcal{O}$. Under the weak field approximation, it is reasonable to consider the potential $\Phi \ll 1$, and thus we have $n \simeq 1$ at the $\mathcal{S}$ and $\mathcal{O}$ points. Let us orient the coordinate system such that the straight line $\overrightarrow{\mathcal{S O}}$ is parallel to the $z$-axis, and assign one of its vertical directions as the $x$-axis. The Fermat's principle demands [13][14],

$$
\left(n \frac{\mathrm{dx}}{\mathrm{d} \ell}\right)_{\mathcal{O}}-\left(n \frac{\mathrm{d} x}{\mathrm{~d} \ell}\right)_{\mathcal{S}}=\int_{\mathcal{S}}^{\mathcal{O}} \mathrm{d} \ell \frac{\partial n}{\partial x}
$$

In the post-Newtonian region, we have ${ }^{2} \Phi \ll 1$, and thus $n_{\mathcal{S}} \simeq n_{\mathcal{O}} \simeq 1$. Thus, the lefthand-side (LHS) of Eq.(3.4) can be replaced by the sum of the two Euclidean geometry angles, $-\left(\theta_{\mathcal{O}}^{\mathrm{E}}+\theta_{\mathcal{S}}^{\mathrm{E}}\right)$, where $\theta_{\mathcal{S}}^{\mathrm{E}}=\left(\frac{\mathrm{d} x}{\mathrm{~d} \ell}\right)_{\mathcal{S}}$ and $\theta_{\mathcal{O}}^{\mathrm{E}}=-\left(\frac{\mathrm{d} x}{\mathrm{~d} \ell}\right)_{\mathcal{O}}$. The Euclidean angles are connected to the measurable angles $\left(\theta_{\mathcal{S}}, \theta_{\mathcal{O}}\right)$ via relation [12], $\tan \left(\theta_{I}\right)=\sqrt{1+2 \Phi} \tan \left(\theta_{I}^{\mathrm{E}}\right)$ where $I=\mathcal{S}, \mathcal{O}$, and $\theta_{\mathcal{S}}\left(\theta_{\mathcal{O}}\right)$ denotes the physical incident (outgoing) angle as shown in Fig. 1. Since $\Phi \ll 1$, we thus expand this relation and deduce $\theta_{I}^{\mathrm{E}} \simeq \theta_{I}$. Then, the LHS of Eq.(3.4) can be further expressed as $-\left(\theta_{\mathcal{O}}^{\mathrm{E}}+\theta_{\mathcal{S}}^{\mathrm{E}}\right) \simeq-\left(\theta_{\mathcal{O}}+\theta_{\mathcal{S}}\right)$. Hence, Eq.(3.4) becomes

$$
-\left(\theta_{\mathcal{O}}+\theta_{\mathcal{S}}\right) \simeq\left(n \frac{\mathrm{d} x}{\mathrm{~d} \ell}\right)_{\mathcal{O}}-\left(n \frac{\mathrm{d} x}{\mathrm{~d} \ell}\right)_{\mathcal{S}}
$$

The deflection angle $\vec{\alpha}$ is the difference between the directions of the incident ray at the point $\mathcal{S}$ and the outgoing ray at the point $\mathcal{O}, \vec{\alpha}=\vec{\theta}_{\mathcal{O}}-\vec{\theta}_{\mathcal{S}}$, with $\left(\vec{\theta}_{\mathcal{S}}, \vec{\theta}_{\mathcal{O}}, \vec{\alpha}\right)$ defined as (counterclockwise, clockwise, clockwise). In the $x$-z plane, this gives $\alpha=\theta_{\mathcal{S}}+\theta_{\mathcal{O}}$ as shown by Fig. 1, where $\left(\theta_{\mathcal{S}}, \theta_{\mathcal{O}}, \alpha\right)=\left(\left|\vec{\theta}_{\mathcal{S}}\right|,\left|\vec{\theta}_{\mathcal{O}}\right|,|\vec{\alpha}|\right)$. The same relation holds for the $y$ - $z$ plane.

\footnotetext{
${ }^{2}$ For instance, in the relevant lensing region around $r=(0.02-2) r_{\text {cri }}$ and for $w \simeq-1$, we can estimate $\Phi \lesssim(0.05-1) \times 10^{-3} \ll 1$, for typical galaxies or galaxy clusters with masses $M \lesssim 10^{16} M_{\odot}$.
} 


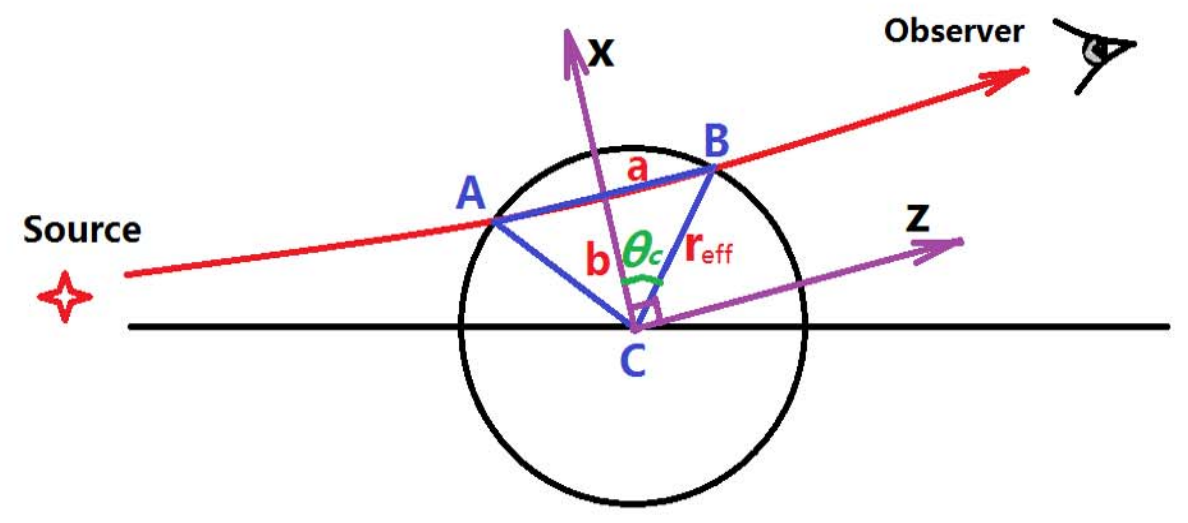

Figure 2. Concave gravitational lensing. The red curve represents the projected trajectory of a ray in the $x$ - $z$ plane, which is deflected by the gravitational potential of dark energy. The circle region defines this lensing as an isolated gravitational system, with radius $r_{\text {eff }}$ and its center at the center of mass $(C)$. The light ray path in the circle is well approximated by a straight line segment $\overline{A B}$, as the intersection between the path and the circle. The shortest distance between $\overline{A B}$ and the center of mass $(C)$ is denoted as $b$. The length $a=\frac{1}{2}|A B|$, and the angle $\theta_{c}=\frac{1}{2} \angle A C B$.

In general, for any similar plane, by substituting Eq.(3.5) into Eq.(3.4), we have

$$
\vec{\alpha}=\vec{\theta}_{\mathcal{O}}-\vec{\theta}_{\mathcal{S}}=-2 \int_{\mathcal{S}}^{\mathcal{O}} \mathrm{d} \ell \nabla_{\perp} \Phi_{\mathrm{N}}-2 \int_{\mathcal{S}}^{\mathcal{O}} \mathrm{d} \ell \nabla_{\perp} \Phi_{\mathrm{DE}}
$$

where $\nabla_{\perp}$ denotes the projection of derivative $\nabla$ onto the plane perpendicular to the ray's path $\ell$.

Consider the matter part having mass $M$ with its mass density $\rho(\mathbf{x})$ distributed over a space region $\Omega\left(\mathbf{x}_{c}\right)$, where $\mathbf{x}_{c}$ is the position of its center of mass. Thus, the total mass of matter is $M=\int_{\Omega} \mathrm{d}^{3} \mathbf{x}^{\prime} \rho\left(\mathbf{x}^{\prime}\right)$. With this, we may express the Newtonian potential $\Phi_{\mathrm{N}}$ as

$$
\Phi_{\mathrm{N}}=-\int_{\Omega} \mathrm{d}^{3} \mathbf{x}^{\prime} \frac{\rho\left(\mathbf{x}^{\prime}\right)}{\left|\mathbf{x}-\mathbf{x}^{\prime}\right|},
$$

where the size of space $\Omega\left(\mathbf{x}_{c}\right)$ is taken to be much smaller than $\left|\mathbf{x}-\mathbf{x}_{c}\right|$, and thus $\left|\mathbf{x}-\mathbf{x}^{\prime}\right| \simeq$ $\left|\mathbf{x}-\mathbf{x}_{c}\right|$. Under the Newtonian potential $\Phi_{\mathrm{N}}$, the deflection angle $\vec{\alpha}_{\mathrm{M}}$ of rays is determined by the matter contribution,

$$
\vec{\alpha}_{\mathrm{M}}=-2 \int_{\mathcal{S}}^{\mathcal{O}} \mathrm{d} \ell \nabla_{\perp} \Phi_{\mathrm{N}} \simeq-2 M \int_{\mathcal{S}}^{\mathcal{O}} \mathrm{d} \ell \frac{\left(\mathbf{x}-\mathbf{x}_{c}\right)_{\perp}}{\left|\mathbf{x}-\mathbf{x}_{c}\right|^{3}} .
$$

The minus sign in front of Eq.(3.8) is notable, and means that the deflecting direction is opposite to $\left(\mathbf{x}-\mathbf{x}_{c}\right)_{\perp}$. Hence, the Newtonian potential tends to trap light rays. This shows that the Newtonian gravitational potential behaves as a convex lens.

Figure 2 illustrates a case where a ray passes through a region within which the dark energy may dominate over the matter. Here, the circle region defines this lensing as an isolated gravitational system (within which the tidal effect from any other gravitational system can be ignored). The circle has its center located at the center of mass of the matter 


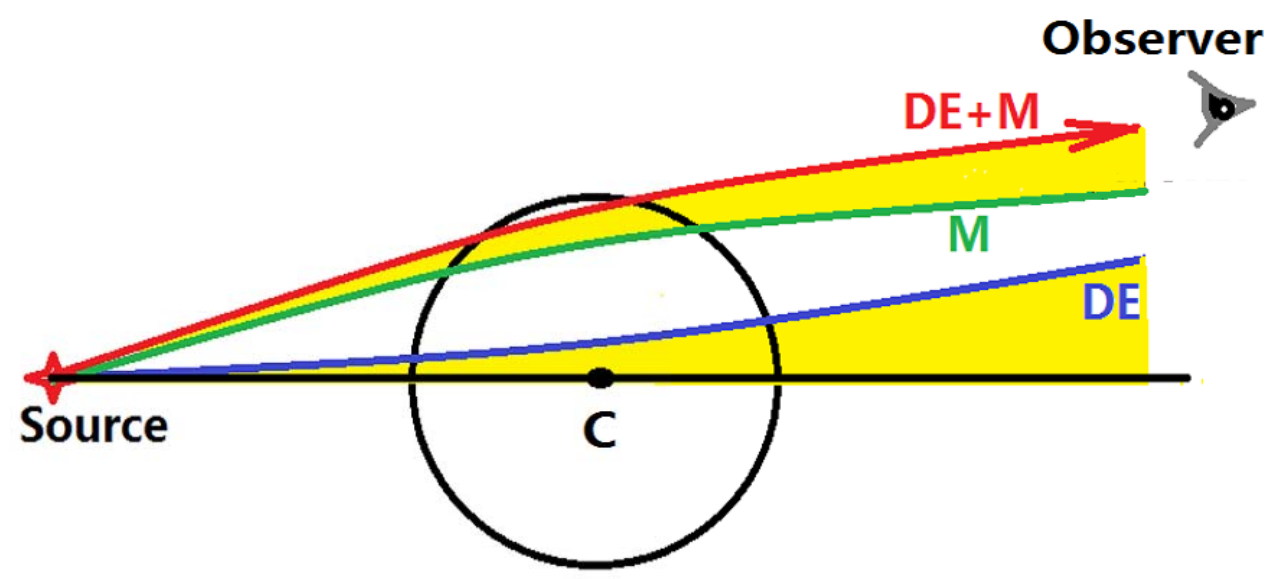

Figure 3. Gravitational lensing, including both contributions by the dark energy and matter. The red curve represents the path of a ray. The green curve stands for the matter contribution alone, and the blue curve illustrates the dark energy contribution alone. The yellow region between the red and green curves shows the deflection effect of the dark energy in the combined path.

$(C)$, and has an effective radius $r_{\text {eff }}$ which is characetrized by the critical radius $r_{\text {cri }}$ (at which the dark energy repulsive force just cancels the attractive Newtonian force, cf. Sec.4). The lensing parameter $b$ is the shortest distance between the incident ray's path $\overline{A B}$ and the point $C$. For a given dark energy potential, denote its center by $\mathbf{x}_{c}$, which coincides with the center of mass $(C)$. From Eq.(2.2), we express the generic dark energy potential as

$$
\Phi_{\mathrm{DE}}(r)=-\left(\frac{r_{o}^{2}}{\left|\mathbf{x}-\mathbf{x}_{c}\right|^{2}}\right)^{\frac{3 w+1}{2}} .
$$

Thus, we have $\nabla_{\perp} \Phi_{\mathrm{DE}}=-(3 w+1) \frac{\left(\mathbf{x}-\mathbf{x}_{c}\right)_{\perp}}{\left|\mathbf{x}-\mathbf{x}_{c}\right|^{2}} \Phi_{\mathrm{DE}}$. Substituting this formula into Eq.(3.6), we derive the dark energy contribution to the deflection angle,

$$
\begin{aligned}
\Delta \vec{\alpha}_{\mathrm{DE}} & =-2 \int_{\mathcal{S}}^{\mathcal{O}} \mathrm{d} \ell \nabla_{\perp} \Phi_{\mathrm{DE}} \\
& =2(-3 w-1) r_{o}^{3 w+1} \int_{\mathcal{S}}^{\mathcal{O}} \mathrm{d} \ell \frac{\left(\mathbf{x}-\mathbf{x}_{c}\right)_{\perp}}{\left|\mathbf{x}-\mathbf{x}_{c}\right|^{3 w+3}} .
\end{aligned}
$$

This deflection angle $\Delta \vec{\alpha}_{\mathrm{DE}}$ is induced by the dark energy potential (3.9) alone. Note that the coefficient of Eq.(3.10) is positive due to $-(3 w+1)>0$, as required by the accelerated expansion of the Universe [15]. This shows that dark energy deflects rays in the same direction as $\left(\mathbf{x}-\mathbf{x}_{c}\right)_{\perp}$. Hence, the dark energy potential tends to diffuse the rays and behaves as a concave lens.

In Fig. 3, we illustrate that gravitational lensing measures the combined effect of two forces: the attractive force induced by matter and the repulsive force induced by dark energy. The deflection angle is co-determined by these two forces with opposite effects,

$$
\vec{\alpha}=\vec{\alpha}_{\mathrm{M}}+\Delta \vec{\alpha}_{\mathrm{DE}}
$$

For the projections onto any given $x$ axis, we have

$$
\operatorname{sign}\left(\Delta \alpha_{\mathrm{DE}}^{x}\right)=-\operatorname{sign}\left(\alpha_{\mathrm{M}}^{x}\right)
$$


which means that the rays are deflected by matter and dark energy in the opposite directions. The dark energy always acts as a concave lens and diffuses the rays, while the matter behaves as a convex lens and attracts the rays. Fig. 3 shows that the Newtonian potential of matter acts as a convex lens and deflects the incident ray along the green curve. On the other hand, the dark energy potential will diffuse the ray along the blue curve. The combined effect is to deflect the ray along the path of the red curve. The net effect induced by dark energy is the shift region (marked in yellow color) between the red curve and the green curve. Measuring this effect can provide a direct evidence for dark energy.

The deflection angle in Eq.(3.10) takes a general form, where the equation of state parameter $w$ plays an important role and can describe different models of dark energy when it takes their corresponding values (cf. Appendix-A). Hence, making precision measurement on the deflection effect (3.10) via gravitational lensing can directly probe the existence of dark energy, and will further discriminate the equation of state parameter $w$ among different dark energy models.

We can follow the standard procedure to write down the lens equation. The angular diameter distances from the source $(\mathcal{S})$ to the observer $(\mathcal{O})$, from the source $(\mathcal{S})$ to the lens $(C)$, and from the lens $(C)$ to the observer $(\mathcal{O})$ are denoted as $D_{\mathcal{S O}}, D_{\mathcal{S} C}$, and $D_{C \mathcal{O}}$ in the FLRW spacetime, respectively. Considering the large scales $D_{\mathcal{S O}}, D_{\mathcal{S C}}, D_{C \mathcal{O}} \gg r_{\text {cri }}$ and the small-angle relations, we have the lens equation,

$$
\vec{\theta} \simeq \vec{\beta}+\vec{\alpha} \frac{D_{\mathcal{S C}}}{D_{\mathcal{S O}}}=\vec{\beta}+\left(\vec{\alpha}_{\mathrm{M}}+\Delta \vec{\alpha}_{\mathrm{DE}}\right) \frac{D_{\mathcal{S} C}}{D_{\mathcal{S O}}},
$$

where the angle $\vec{\beta}$ corresponds to the position of the unlensed source $\mathcal{S}$, the angle $\vec{\theta}$ corresponds to the apparent position, and the deflection angle $\vec{\alpha}=\vec{\alpha}_{\mathrm{M}}+\Delta \vec{\alpha}_{\mathrm{DE}}$ is given by Eq.(3.11). As we will show in Sec.4-5 as well as Appendices A.4 and C.2, this deflection angle $\vec{\alpha}$ remains unchanged for the regions outside the deflection region. The form of the lens equation (3.13) agrees to Refs. [4][16]. It is clear that in Eq.(3.13), the matter and dark energy contributions to the deflection angle $\vec{\alpha}\left(=\vec{\alpha}_{\mathrm{M}}+\Delta \vec{\alpha}_{\mathrm{DE}}\right)$ are on equal footing. Hence, the dark energy deflection $\vec{\alpha}_{\mathrm{DE}}$ cannot be attributed to the angular diameter distances separately while leaving the matter contribution $\vec{\alpha}_{\mathrm{M}}$ unaffected. This point was also mentioned in [17] for the case of $w=-1$.

Before concluding this section, we comment on the special limit $M \rightarrow 0$. In this limit, the lensing setup is removed, while the dark energy remains everywhere in the Universe. So one may wonder whether the dark energy potential $\Phi_{\mathrm{DE}}$ could still deflect any incident ray passing through the space. The answer is no, and the explanation is instructive. Inspecting the gravitational potential $\Phi_{\mathrm{N}}+\Phi_{\mathrm{DE}}$ in Eq.(2.2) and the spacetime metric (2.1) or (A.18), we see that the origin point of the coordinate frame for this isolated lensing system is chosen to be the center of mass point $C$ of $M$ (cf. Figs. 2-3), and the potential $\Phi_{\mathrm{N}}+\Phi_{\mathrm{DE}}$ is consistently solved from the Einstein equation (A.2) as shown in Appendix A.1. But, in the limit $M \rightarrow 0$, 
the current center of mass point $C$ no longer has its meaning. Hence, we have to redefine the isolated system by including the source of this incident ray, where the source must have a nonzero mass, say $M^{\prime}$, whose center of mass point may be denoted as $C^{\prime}$. Now, for the consistency of solving Einstein equation, we must redefine a new coordinate frame with its origin at the point $C^{\prime}$, and then rederive the potential $\Phi_{\mathrm{N}}+\Phi_{\mathrm{DE}}$ in this frame as Appendix A.1. The key point is to note: because the incident ray is emitted from its source $M^{\prime}$ centered at $C^{\prime}$, it propagates along the radial direction of the new potential form $\Phi_{\mathrm{N}}+\Phi_{\mathrm{DE}}$. Hence, no deflection on this ray could be generated according to the definition (3.6), and we can directly infer the deflection angle from Eqs.(3.8) and (3.10), $\vec{\alpha}_{\mathrm{M}}=\Delta \vec{\alpha}_{\mathrm{DE}}=0$. This shows that the current formulation is fully consistent.

\section{Probing Concave Lensing of Dark Energy}

When a ray passes through the deflection region, the gravitational field can bend the ray by a small deflection angle. The ray's path $\ell$ over the deflection region may be approximated by a straight line segment $\overline{A B}$, as in Fig. 2. For convenience, we choose a new coordinate system in Fig. 2, where the $z$-axis is parallel to the straight line $\overline{A B}$. Here we only show the lensing effect for dark energy. In this coordinate system, the point on the line segment $\overline{A B}$ is denoted as $z=z(\ell)$. From Eq.(3.10), we have

$$
\Delta \vec{\alpha}_{\mathrm{DE}}=2(-3 w-1) r_{o}^{3 w+1}\left(\mathbf{x}-\mathbf{x}_{c}\right)_{\perp} \int_{A}^{B} \frac{\mathrm{d} z}{\left|\mathbf{x}-\mathbf{x}_{c}\right|^{3 w+3}} .
$$

To simplify the analysis, we assume that the deflector is an isolated and spherical system, in which all the matter can be approximated as a spherically symmetric mass-distribution with total mass $M$. The gravitational potential is described by Eq.(2.2). The recent combination of BAO, SN and CMB data gives [15], $w=-0.97 \pm 0.05$ for $w \mathrm{CDM}$ (constant $w$ with a flat universe), which is fairly close to $w=-1$ and leads to $3 w+1<0$ at $12.7 \sigma$ level and $3 w+2<0$ at $6.1 \sigma$ level. Thus, Eq.(2.2) shows that the dark energy force is always repulsive [cf. Eq.(A.20)] and increases with the distance $r$. When $r$ reaches a critical value $r_{\text {cri }}$, this repulsive force will balance the attractive Newtonian force, where the net force acting on matter vanishes. Hence, beyond $r_{\text {cri }}$ the matter will begin to escape the gravitational bonds. From the gravitational potential (2.2), we deduce both the Newtonian force and dark energy force as in Eq.(A.20) of Appendix A.1. Hence, we can derive the critical radius $r_{\text {cri }}$ (for a generical state parameter $w)$,

$$
r_{\text {cri }}=r_{o}\left(\frac{M}{|3 w+1| r_{o}}\right)^{-\frac{1}{3 w}}
$$

at which the dark energy force balances the Newtonian attraction. For the special case of $w=-1$, Eq. (4.2) reduces to $r_{\text {cri }}=(3 M / \Lambda)^{1 / 3}$, which coincides with [18].

In the region $r \ll r_{\text {cri }}$, the gravitational force is dominated by the Newtonian attraction of matter. In the region with $r>r_{\text {cri }}$, the repulsive dark energy force will dominate over 
the Newtonian attraction. The post-Newtonian approximation holds well for $r \lesssim r_{\text {cri }}$. The weak field approximation requires the effective radius $r_{\text {eff }} \ll r_{o}$. Also, the size of $r_{\text {eff }}$ is properly chosen to avoid tidal effect from any other gravitational object, so that the lensing system behaves as an isolated system. To have nonnegligible dark energy effect on the lensing, we consider $r_{\text {eff }}$ around the same order of $r_{\text {cri }}$, i.e., the ratio $n_{\text {eff }} \equiv r_{\text {eff }} / r_{\text {cri }}=O(1)$. In the region with $r_{\text {cri }} \lesssim r \lesssim r_{\text {eff }}$, one may still make estimates under the post-Newtonian approximation.

The spacetime region $r \lesssim r_{\text {eff }}$ of this isolated astrophysical system can be smoothly embedded into a nearly flat FLRW universe $\left(r>r_{\text {eff }}\right)$ [19][20]. Thus, we require that the matter density of such an isolated lensing system equals the averaged matter density $\rho_{M}$ at cosmological scales from the CMB measurements. This will also ensure the Einstein equation to hold across the boundary $\left(r=r_{\text {eff }}\right)$ [19][20]. Hence, we have the matching condition

$$
\frac{M}{\frac{4 \pi}{3} r_{\mathrm{eff}}^{3}}=\rho_{M},
$$

where we have [1], $\rho_{M}=\rho_{\mathrm{DE}}\left(\Omega_{\mathrm{M}} / \Omega_{\mathrm{DE}}\right) \simeq 0.44 \rho_{\mathrm{DE}}$. From Eqs.(4.3) and (4.2), we derive

$$
r_{\text {eff }}=\left(\frac{3 M}{4 \pi \rho_{M}}\right)^{\frac{1}{3}}=r_{\text {cri }}\left(\frac{3|3 w+1|}{4 \pi r_{o}^{2} \rho_{M}}\right)^{\frac{1}{3}}\left(\frac{r_{\text {cri }}}{r_{o}}\right)^{|w|-1} \text {. }
$$

We may give an explicit estimate on the size of the effective radius $r_{\text {eff }}$. For the case of the cosmological constant as dark energy $(w=-1)$, we derive the dark energy density $\rho_{\mathrm{DE}}=\rho_{\Lambda}$ as follows,

$$
\rho_{\Lambda}=\frac{\Lambda}{8 \pi}=\frac{3 M}{8 \pi r_{\mathrm{cri}}^{3}} .
$$

Thus, using Eqs.(4.4)-(4.5) with $w=-1$, we can estimate the size of $r_{\text {eff }}$ in terms of the critical radius $r_{\text {cri }}$,

$$
r_{\text {eff }}=r_{\text {cri }}\left(\frac{2}{\Omega_{\mathrm{M}} / \Omega_{\Lambda}}\right)^{\frac{1}{3}} \simeq 1.7 r_{\mathrm{cri}},
$$

and $n_{\text {eff }}=r_{\text {eff }} / r_{\text {cri }} \simeq 1.7=O(1)$, as expected. This is a fairly reasonable estimate. The effective radius $r_{\text {eff }}$ may serve to characterize the transition scale between the Schwarzschildde Sitter (SdS) spacetime inside the deflection region $\left(r \lesssim r_{\text {eff }}\right)$ and the FLRW spacetime beyond the lensing system $\left(r>r_{\text {eff }}\right)$. We will further discuss the embedding of the isolated lensing system into the flat FLRW spacetime in Appendices A.4 and C.2.

According to Fig. 2, we can express the lensing parameters $(a, b)$ as, $a=r_{\text {eff }} \sin \theta_{c}$ and $b=\left|\left(\mathbf{x}-\mathbf{x}_{c}\right)_{\perp}\right|=r_{\text {eff }} \cos \theta_{c}$. Thus, from Eq.(4.1), we compute the size of the deflection angle produced by dark energy, $\Delta \alpha_{\mathrm{DE}}=\left|\Delta \vec{\alpha}_{\mathrm{DE}}\right|$,

$$
\begin{aligned}
\Delta \alpha_{\mathrm{DE}} & =2|3 w+1| r_{o}^{3 w+1} b \int_{-a}^{+a} \frac{\mathrm{d} z}{\left(b^{2}+z^{2}\right)^{(3 w+3) / 2}} \\
& =4|3 w+1|\left(\frac{r_{o}}{r_{\text {eff }}}\right)^{3 w+1} \int_{0}^{\theta_{c}} \mathrm{~d} \theta\left(\frac{\cos \theta}{\cos \theta}\right)^{3 w+1}
\end{aligned}
$$


where $\theta_{c}=\arctan (a / b)<\frac{\pi}{2}$. Along the same path, from Eq.(3.8), we compute the size of matter-induced deflection angle $\alpha_{\mathrm{M}}=\left|\vec{\alpha}_{\mathrm{M}}\right|$ as follows,

$$
\alpha_{\mathrm{M}}=\frac{4 M}{r_{\mathrm{eff}}} \int_{0}^{\theta_{c}} \mathrm{~d} \theta \frac{\cos \theta}{\cos \theta_{c}}=\frac{4 M}{r_{\text {eff }}} \tan \theta_{c} .
$$

For the special case of fixing $b\left(=r_{\text {eff }} \cos \theta_{c}\right)$ and taking $r_{\text {eff }} \rightarrow \infty$ (with $\left.\theta_{c} \rightarrow \frac{\pi}{2}\right)$, we see that the formula (4.8) recovers the conventional result of light bending [21][10], $\alpha_{\mathrm{M}}=\frac{4 M}{b}$. Hence, the size of the total deflection angle is $\alpha=|\vec{\alpha}|=\left|\vec{\alpha}_{M}+\Delta \vec{\alpha}_{D E}\right|$. From Eq.(3.12), we see that the dark energy contribution always tends to cancel the matter contribution to the deflection angle $\alpha$, and will produce a deficit as compared to the conventional lensing prediction without dark energy.

As an explicit demonstration, let us consider the cosmological constant model of dark energy. It corresponds to $w=-1$ and $r_{o}=\sqrt{6 / \Lambda}$ in the gravitational potential (2.2). From Eq.(4.2), the lensing system in this case has a critical radius $r_{\text {cri }}=(3 M / \Lambda)^{1 / 3}$. For the region within the effective radius $r \lesssim r_{\text {eff }} \sim r_{\text {cri }}$, the post-Newtonian approximation holds well. With Eq.(4.7), we compute the dark energy contribution to the deflection angle,

$$
\Delta \alpha_{\mathrm{DE}}=4\left(\frac{r_{\mathrm{eff}}}{r_{o}}\right)^{2} \sin 2 \theta_{c}=2 n_{\mathrm{eff}}^{2} \sin 2 \theta_{c}\left(\frac{\Lambda M^{2}}{3}\right)^{\frac{1}{3}},
$$

where $n_{\text {eff }}=r_{\text {eff }} / r_{\text {cri }}=O(1)$. The maximal value $\Delta \alpha_{\mathrm{DE}}^{\max }=4\left(r_{\text {eff }} / r_{o}\right)^{2}=2 n_{\text {eff }}^{2}\left(\Lambda M^{2} / 3\right)^{1 / 3}$ is achieved at $\theta_{c}=\frac{\pi}{4}$.

We further clarify the special limit of $M \rightarrow 0$. From the expression $r_{\text {cri }}=(3 M / \Lambda)^{1 / 3}$, we find that the limit $M \rightarrow 0$ leads to $r_{\text {eff }} \sim r_{\text {cri }} \propto M^{\frac{1}{3}} \rightarrow 0$. Thus, Eq.(4.9) gives $\Delta \alpha_{\mathrm{DE}} \propto r_{\text {eff }}^{2} \propto M^{\frac{2}{3}} \rightarrow 0$ for each given angle $\theta_{c}$. For a general state parameter $w$, we can deduce from Eqs.(4.7) and (4.2), $\Delta \alpha_{\mathrm{DE}} \propto r_{\text {eff }}^{-(3 w+1)} \propto r_{\text {cri }}^{-(3 w+1)} \propto M^{\left(1+\frac{1}{3 w}\right)}$, in which $1+\frac{1}{3 w}>0$ holds at $12.7 \sigma$ level as required by the existing observational data [15]. Hence, the zero mass limit $M \rightarrow 0$ always enforces $\Delta \alpha_{\mathrm{DE}} \rightarrow 0$. This also agrees to our conclusion at the end of Sec. 3, and shows that our approach is fully consistent.

For the current case of single lensing and in the regions beyond the isolated lensing system with $r^{3} \gg r_{\text {eff }}^{3} \sim r_{\text {cri }}^{3}$, the Newtonian potential becomes negligible, and we explicitly show in Appendices A.4 and C.2 that the spacetime geometry conformally recovers the FLRW metric with vanishing spatial curvature $(K=0)$, which will not change the deflection angle of the passing light ray. Hence, the total lensing effect of dark energy is given by Eq. (4.9).

From Eqs.(4.8) and (4.9), we further derive the ratio,

$$
\frac{\Delta \alpha_{\mathrm{DE}}}{\alpha_{\mathrm{M}}}=n_{\mathrm{eff}}^{3} \cos ^{2} \theta_{c}=n_{\mathrm{eff}} \frac{b^{2}}{r_{\mathrm{cri}}^{2}}
$$

where $n_{\text {eff }}=r_{\text {eff }} / r_{\text {cri }}=O(1)$. This means that when the lensing parameters $\left(b, r_{\text {eff }}\right)$ are comparable to the critical radius $r_{\text {cri }}$, the dark energy correction $\Delta \alpha_{\mathrm{DE}}$ will be significant as compared to the matter contribution $\alpha_{\mathrm{M}}$. Hence, for the case of $b, r_{\text {eff }} \sim r_{\text {cri }}$, measuring 
gravitational lensing effect can directly probe the dark energy via its produced deficit in the deflection angle $\alpha=\left|\vec{\alpha}_{\mathrm{M}}+\Delta \vec{\alpha}_{\mathrm{DE}}\right|$.

The cosmological constant model of dark energy corresponds to $w=-1$ and $r_{o}=$ $\sqrt{6 / \Lambda}$ in Eq. (2.2). Thus, we can estimate $r_{o} \simeq 0.72 \times 10^{4} \mathrm{Mpc}$. For an astrophysical object, such as galaxy or galaxy cluster, its typical mass is about $10^{12-16} M_{\odot}$, where $M_{\odot}$ is the solar mass. We estimate its critical radius $r_{\text {cri }}=(3 M / \Lambda)^{1 / 3} \simeq(1.1-23) \mathrm{Mpc}$. The effective radius $r_{\text {eff }}=n_{\text {eff }} r_{\text {cri }}$, where the coefficient $n_{\text {eff }}=O(1)$ depends on the definition of the lens as an isolated system. For an ideal isolated lens, our matching estimate (4.6) gives $n_{\mathrm{eff}} \simeq 1.7$. Thus, from Eq.(4.9), we estimate the dark energy contribution, $\Delta \alpha_{\mathrm{DE}}^{\max } \simeq n_{\mathrm{eff}}^{2} \times\left(0.018^{\prime \prime}-8.5^{\prime \prime}\right)$. This is already a significant effect, as we may recall that the conventional prediction of the matter-induced light bending through the sun is $\alpha_{\odot}=1.75^{\prime \prime}$ [21][10].

The current lensing experiments for galaxies at redshift $z=0.1-0.5$ [22][23] can measure the deflection angle to a relative accuracy of $\Delta \alpha / \alpha \sim 5 \%$, and measure $\Delta \alpha \sim 0.01^{\prime \prime}$. For galaxies or galaxy clusters at redshift $z=0.5-2$ [24-26], the current measurements can reach $\Delta \alpha / \alpha \sim 5 \%$ and $\Delta \alpha \sim 0.05^{\prime \prime}$. For the actual experimental measurements, a $5 \%$ accuracy of $\alpha$ is expected to be on the conservative side, as we will choose in Fig. 4 for comparison.

In passing, we also note that Ishak et al [16] independently considered some typical lensing systems with galaxy cluster masses $M=(1.37-13.8) \times 10^{13} M_{\odot}$, and used a different method (formula) to estimate the cosmological constant contribution to the light bending angle, $\Delta \alpha_{\Lambda} \simeq\left(0.005^{\prime \prime}-0.025^{\prime \prime}\right)$. For the conventional matter-induced light bending angle $\alpha_{\mathrm{M}}$ at the leading order, Ref.[16] agrees to our Eq.(4.8) with its distance $R$ (between the centers of the deflection region and the lens) related to our lensing parameters via $R=$ $r_{\text {eff }} / \tan \theta_{c}$. For the above mass-range of typical lenses, they chose the deflection region with $R \simeq(0.06-0.14) \mathrm{Mpc} \simeq(0.02-0.03) r_{\text {cri }} \ll r_{\text {eff }} \sim r_{\text {cri }}$. Since we choose the deflection region with $r \leqslant r_{\text {eff }}=O\left(r_{\text {cri }}\right)$ and our Eq.(4.9) gives $\Delta \alpha_{\mathrm{DE}} \propto r_{\text {eff }}^{2} \sim r_{\text {cri }}^{2}$, it is expected that for $r_{\text {eff }}=r_{\text {cri }}(\gg R)$, we obtain a much larger bending angle $\Delta \alpha_{\mathrm{DE}}^{\max } \simeq\left(0.11^{\prime \prime}-0.49^{\prime \prime}\right)$ for the same lensing mass-range of [16]. If we choose the effective radius $r_{\text {eff }}$ around its lower limit $r_{\text {eff }} \simeq 0.3 r_{\text {cri }}$ (cf. the discussion below), this means a smaller deflection region and we deduce a smaller bending angle accordingly, $\Delta \alpha_{\mathrm{DE}}^{\max } \simeq\left(0.009^{\prime \prime}-0.044^{\prime \prime}\right)$.

In Fig. 4, we illustrate the dark energy contribution $\Delta \alpha_{\mathrm{DE}}$ to the deflection angle $\alpha$ of the gravitational lensing. To be concrete, we realize the dark energy via cosmological constant. Plot (a) presents the ratio of $\Delta \alpha_{\mathrm{DE}}$ over the matter contribution $\alpha_{\mathrm{M}}$, while plot (b) depicts the relative ratio $\Delta \alpha_{\mathrm{DE}} / \alpha$. Using Eqs.(4.9) and (4.10), we vary the ratio $n_{\text {eff }}=r_{\text {eff }} / r_{\text {cri }}$ for the horizontal axis, and choose $b / r_{\text {cri }}=(0.3,0.4,0.5,0.6)$ as the benchmark points, corresponding to the curves from bottom to top in each plot. For the shaded region of $r_{\text {eff }} / r_{\text {cri }}<0.3$, the matter sector could not well behave as an isolated gravitational system, so it will be excluded from the analysis. In the deflection region of $0.3 r_{\text {cri }} \lesssim r \lesssim r_{\text {eff }}$, 

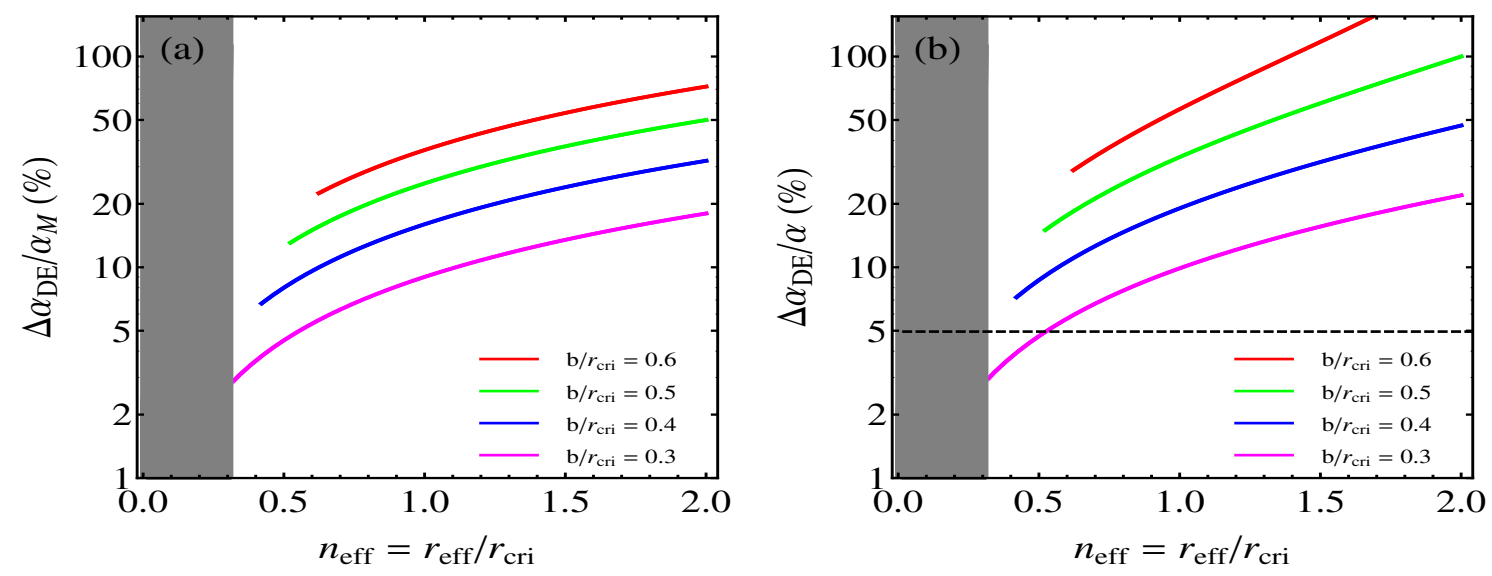

Figure 4. Dark energy contribution $\Delta \alpha_{\mathrm{DE}}$ to the deflection angle $\alpha$ of the gravitational lensing. Plot (a) shows $\Delta \alpha_{\mathrm{DE}}$ relative to the matter contribution $\Delta \alpha_{\mathrm{M}}$, while plot (b) depicts the ratio $\Delta \alpha_{\mathrm{DE}} / \alpha$. An accuracy of $5 \%$ on the $\alpha$ measurement can be reached by the current lensing experiments [24-26], as illustrated by the horizontal dashed line in plot (b).

there is almost no matter distribution and the Newtonian potential of matter can be well approximated as generated by a point-like object. For instance, the Milky Way has its dark matter halo extended out to the galactocentric distance of $\sim 200 \mathrm{kpc}$ [27], which is about $0.2 r_{\text {cri }}$ for $r_{\text {cri }} \approx 10^{3} \mathrm{kpc}$. So it is safe to choose a larger distance $\sim 0.3 r_{\text {cri }}$ as the lower limit of effective radius $r_{\text {eff }}$. In the deflection region of $0.3 r_{\text {cri }} \lesssim r \lesssim r_{\text {eff }}=O\left(r_{\text {cri }}\right)$, the effect of dark energy contribution may be extracted by subtracting the background of matter contribution. This subtraction does not depend on the detail of matter distribution since it behaves point-like. Taking an accuracy of $5 \%$ for the present lensing measurements, we see that in the region of $n_{\text {eff }}>0.3$, the predicted curves in plot (b) are mostly above the dashed black horizontal line (denoting the $5 \%$ accuracy), and are thus testable.

We note that the dark energy lensing effect (4.7) is fully expressed in terms of the dark energy parameters $\left(w, r_{o}\right)$ besides the lensing parameters $r_{\text {eff }}$ and $\theta_{c}=\arccos \left(b / r_{\text {eff }}\right)$. Hence, Eq.(4.7) holds for both the cosmological constant model $(w=-1)$ and other dark energy models, such as the quitessence model $\left(-1<w<-\frac{1}{3}\right)$ and the phantom model $(w<-1)$. Thus, making precision lensing measurements is important for probing the dark energy contribution $\Delta \alpha_{\mathrm{DE}}$ to the deflection angle, and for further discriminating dark energy models with different values of the state parameter $w$. Eq.(4.7) shows that for a given gravitational lensing system, the dark energy correction $\Delta \alpha_{\mathrm{DE}}$ is fully determined by the two generic parameters $\left(w, r_{o}\right)$. So it is possible to fit $\left(w, r_{o}\right)$ from analyzing the variation of luminance or distortion of the $2 \mathrm{~d}$ image of background light sources due to the concave lensing of dark energy. With extracted information about the state parameter $w$, we could identify the proper model of dark energy. The current telescopes appear sensitive to probing this parameter via gravitational lensing. 


\section{Light Orbital Equation with Generic State Parameter w}

In this section, considering a gravitational lensing system of total mass $M$ (with a pointlike spherical mass-distribution), we first derive the light orbital equation for dark energy models with a generic state parameter $w$ in the $\operatorname{SdS} w$ spacetime. For the special case of the cosmological constant model of dark energy $(w=-1)$, it reduces to the light orbital equation in the familiar SdS spacetime. At the end of this section, we also clarify the difference of our approach from the literature concerning the light bending effect induced by $\Lambda$.

From the metric (A.18) in Appendix A.1 and using the null geodesic condition $\mathrm{d} S^{2}=0$, we derive the following light orbital equation with a generic state parameter $w$,

$$
\left(\frac{\mathrm{d} u}{\mathrm{~d} \phi}\right)^{2}=\frac{1}{b^{2}}-u^{2}+2 M u^{3}+2 r_{o}^{3 w+1} u^{3(w+1)},
$$

for $\theta=\frac{\pi}{2}$. In the above, $u=1 / r, b=L / E, b$ is the impact parameter, and $E$ and $L$ are total energy and angular momentum, respectively. We will give the derivation of Eq.(5.1) in Appendix B. The cosmological constant model of dark energy predicts $w=-1$, and in this case our lensing system corresponds to the SdS spacetime. We note that for $w=-1$, the last term on the right-hand-side of Eq.(5.1) becomes a pure constant, independent of $r$. Thus, we can reexpress Eq.(5.1) as follows,

$$
\left(\frac{\mathrm{d} u}{\mathrm{~d} \phi}\right)^{2}=\frac{1}{b^{2}}+\frac{2}{r_{o}^{2}}-u^{2}+2 M u^{3}+\frac{2}{r_{o}^{2}}\left[\left(r_{o} u\right)^{3(w+1)}-1\right]
$$

where the last term on the right-hand-side vanishes for the cosmological constant dark energy $(w=-1)$, and gives nonzero $r$-dependent contribution for other dark energy models with $w \neq-1$.

From Eq.(5.1), we can derive a second-order ordinary differential equation (ODE),

$$
\frac{\mathrm{d}^{2} u}{\mathrm{~d} \phi^{2}}=-u+3 M u^{2}+3(w+1) r_{o}^{3 w+1} u^{3 w+2},
$$

which holds for a general state parameter $w$. Under the weak deflection assumption, the orbital equation can be approximated by introducing a small perturbation to the undeflected straight line in the flat space. In Eq.(B.8), the $w$-dependent terms arise from the correction of dark energy, which vanishes for $w=-1$. It shows that only for the special case of the cosmological constant $(w=-1)$, Eq.(B.8) reduces to the following form,

$$
\frac{\mathrm{d}^{2} u}{\mathrm{~d} \phi^{2}}=-u+3 M u^{2},
$$

which agrees to [9] and happens to take the same form as the conventional light orbital equation with $\Lambda=0$.

But, the original orbital equation (5.1) (which is the first-order ODE) does depend on the dark energy term even for the case of $w=-1$,

$$
\left(\frac{\mathrm{d} u}{\mathrm{~d} \phi}\right)^{2}=\frac{1}{b^{2}}-u^{2}+2 M u^{3}+\frac{\Lambda}{3} .
$$


It may be rewritten as [28],

$$
\left(\frac{\mathrm{d} u}{\mathrm{~d} \phi}\right)^{2}=\frac{1}{B^{2}}-u^{2}+2 M u^{3}
$$

with an effective impact parameter $B$ defined via $\frac{1}{B^{2}}=\frac{1}{b^{2}}+\frac{\Lambda}{3}$. But, for all other cases with $w \neq-1$, the $w$-dependent dark energy term in Eq.(5.1) or Eq.(5.2) does depend on the coordinate $r$, and thus cannot be absorbed into the redefinition of an effective impact parameter $B$. This means that making precision measurement on the light deflection can discriminate between dark energy models with $w=-1$ and $w \neq-1$. Furthermore, Eq.(5.6) holds only for the point-like light source, rather than the more realistic 2-dimensional (2d) light source. For such $2 d$ light source, the impact parameter $b$ varies its value for different positions on the surface of the source. Hence, no universal effective impact parameter $B$ exists to fully absorb $\Lambda$ effect in such realistic cases. Thus, fitting the lensing data from $2 \mathrm{~d}$ light sources can discriminate the deflection effect induced by $\Lambda$.

\section{Conclusions}

The mystery of dark energy poses a great challenge to modern science. Various on-going and future experiments [2] are making enormous efforts to probe the origin and nature of dark energy. In this work, we showed that gravitational lensing can serve as an important tool for direct probe of dark energy at astrophysical scales. For an isolated astrophysical system (such as the galaxy or galaxy cluster), we derived a general form for the repulsive potential of dark energy with a generic equation of state parameter $w$, and computed its direct lensing effect under the post-Newtonian approximation. We demonstrated that the dark energy acts as a concave lens, contrary to the convex lensing effect of both visible and dark matter. Hence, measuring this concave lensing effect can directly probe the existence and nature of dark energy.

In section 2, under the post-Newtonian approximation, we presented the repulsive powerlaw potential (2.2) for dark energy, containing a generic equation of state parameter $w$ which can describe various dark energy models. This is derived in the Schwarzschild-de Sitter spacetime with general $w$ (denoted as $\mathrm{SdS} w$ metric). Then, in section 3, with the potential (2.2), we derived the dark energy contribution $\Delta \vec{\alpha}_{\mathrm{DE}}$ to the deflection angle $\vec{\alpha}$ of a gravitational lensing system in Eq.(3.10). We further proved in Eq.(3.12) that this deflection always takes opposite sign to the matter contribution $\vec{\alpha}_{M}$ along any given direction. Hence, the dark energy effect always acts as a concave lens, in contrast with the conventional matter effect, as shown in Fig. 2 and Fig. 3.

In section 4, for a general state parameter $w$, we derived the critical radius $r_{\text {cri }}$ in Eq.(4.2), at which the dark energy force balances the Newtonian attraction. For a given gravitational lensing with mass $M$, we imposed the matching condition (4.3) and deduced 
its effective radius $r_{\text {eff }}$ in Eqs.(4.4) and (4.6), which defines this lens as an ideal isolated system. Eq.(4.6) explicitly shows $r_{\text {eff }}=O\left(r_{\text {cri }}\right)$. Then, we computed the size of the darkenergy-induced deflection angle, $\Delta \alpha_{\mathrm{DE}}$, as given by Eq.(4.7). We demonstrated that the dark-energy-induced deflection angle $\Delta \alpha_{\mathrm{DE}} \propto M^{\left(1+\frac{1}{3 w}\right)}$ (with $1+\frac{1}{3 w}>0$ ), which increases with the lensing mass $M$ and consistently vanishes in the zero-mass limit $M \rightarrow 0$. We explicitly derived the prediction of $\Delta \alpha_{\mathrm{DE}}$ in Eq.(4.9) for the cosmological constant model of dark energy $(w=-1)$. We computed the ratio between the dark energy and matter contributions to the deflection angle in Eq.(4.10). In Fig.4(a)-(b), we presented the relative ratios $\Delta \alpha_{\mathrm{DE}} / \alpha_{\mathrm{M}}$ and $\Delta \alpha_{\mathrm{DE}} / \alpha$, as functions of the effective radius $r_{\text {eff }}$ (in unit of $r_{\text {cri }}$ ) and for different benchmarks of the lensing parameter $b$. We estimated this dark energy lensing effect and found that the current gravitational lensing experiments are already sensitive to the direct probe of dark energy. In section 5, for an isolated lensing system, we presented a new orbital equation (5.1) [or (5.2)] of light rays including the generic state parameter $w$, and discussed its implication for the observations. We gave a systmetical derivation of Eq.(5.1) in Appendix B. We showed in Appendix C.1 that the cosmic expansion effect is negligible within a lensing system at typical astrophysical scales. For regions outside the lensing system, we proved the approximate conformal flatness of the SdS metric and the $\mathrm{SdS} w$ metric for $r^{3} / r_{\text {cri }}^{3} \gg 1$, as in Appendix A.4 and Appendix C.2. So there is no leading correction to the deflection angle studied in Sec. 3-4.

Finally, we conclude that in general it is important to make the precision measurements on the dark-energy-induced deflection effect (4.7) via astrophysical gravitational lensing, which could directly probe the nature of dark energy and further discriminate the equation of state parameter $w$ among different dark energy models.

\section{Acknowledgements}

We thank Xingang Chen, Stephen Hsu, Mustapha Ishak, Yipeng Jing, Mauro Sereno, YuChen Wang, and Jun Zhang for useful discussions. We also thank Xuelei Chen, Zuhui Fan and Pengjie Zhang for related discussions. This research was supported in part by the National NSF of China (under Grants 11275101, 11135003, 11675086).

\section{A Dark Energy Potential and Dark Energy Models}

In Appendix A.1, we first use Einstein equation to derive the generic dark energy potential (2.2) for an isolated astrophysical system. Then, we discuss its connection with the typical dark energy models [11], in Appendix A.2-A.4, including the phantom model, the quintessence model, and the cosmological constant model. In Appendix A.4, we will further show that in the regions beyond the isolated lensing system with $r^{3} / r_{\text {cri }}^{3} \gg 1$, the Newtonian potential becomes negligible, and the spacetime geometry reduces to the de Sitter metric which con- 
formally recovers the flat FLRW metric with vanishing spatial curvature $(K=0)$. So the region $r^{3} / r_{\text {cri }}^{3} \gg 1$ will not change the deflection angle of the passing light ray.

\section{A.1 General Gravitational Potential Including Dark Energy}

The static and spherically symmetric spacetime can be described by the following form,

$$
\mathrm{d} S^{2}=e^{\mathbb{A}} \mathrm{d} t^{2}-e^{\mathbb{B}} \mathrm{d} r^{2}-r^{2}\left(\mathrm{~d} \theta^{2}+\sin ^{2} \theta \mathrm{d} \phi^{2}\right),
$$

where $\mathbb{A}=\mathbb{A}(r)$ and $\mathbb{B}=\mathbb{B}(r)$ are radial functions. For conventions, we choose the Minkowski metric $\eta_{\mu \nu}=\eta^{\mu \nu}=\operatorname{diag}(1,-1,-1,-1)$, and set the constants $G=h=c=1$ throughout as mentioned above Eq.(2.2). Thus, the Einstein equation [21] takes the form

$$
R_{\mu \nu}-\frac{1}{2} g_{\mu \nu} R=-8 \pi T_{\mu \nu},
$$

where the energy-momentum tensor $T_{\mu \nu}$ contains contributions from both matter and dark energy. We can use the metric (A.1) to express the Einstein equation (A.2) as follows,

$$
\begin{aligned}
& 8 \pi T_{t}^{t}=-e^{-\mathbb{B}}\left(\frac{1}{r^{2}}-\frac{\mathbb{B}^{\prime}}{r}\right)+\frac{1}{r^{2}}, \\
& 8 \pi T_{r}^{r}=-e^{-\mathbb{B}}\left(\frac{1}{r^{2}}+\frac{\mathbb{A}^{\prime}}{r}\right)+\frac{1}{r^{2}}, \\
& 8 \pi T_{\theta}^{\theta}=8 \pi T_{\phi}^{\phi}=-\frac{e^{-\mathbb{B}}}{2}\left(\mathbb{A}^{\prime \prime}+\frac{\mathbb{A}^{\prime 2}}{2}+\frac{\mathbb{A}^{\prime}-\mathbb{B}^{\prime}}{r}-\frac{\mathbb{A}^{\prime} \mathbb{B}^{\prime}}{2}\right),
\end{aligned}
$$

where $\mathbb{A}^{\prime}=\mathrm{d} \mathbb{A}(r) / \mathrm{d} r$ and $\mathbb{B}^{\prime}=\mathrm{d} \mathbb{B}(r) / \mathrm{d} r$.

The condition of linearity and additivity requires $T_{t}{ }^{t}=T_{r}{ }^{r}$ [29], which leads to $\mathbb{A}+\mathbb{B}=$ constant. Here, one can fix the gauge of $\mathbb{A}+\mathbb{B}=0$ for the static coordinate system without losing generality, since the constant factor can be absorbed by a proper rescaling of time. Setting $\mathbb{B}=-\ln (1+F)$, we have the following,

$$
\begin{aligned}
4 \pi T_{t}^{t} & =4 \pi T_{r}^{r}=-\frac{1}{2 r^{2}}\left(F+r F^{\prime}\right), \\
4 \pi T_{\theta}^{\theta} & =4 \pi T_{\phi}^{\phi}=-\frac{1}{4 r}\left(2 F^{\prime}+r F^{\prime \prime}\right) .
\end{aligned}
$$

The condition of linearity and additivity means that the linear sum of the possible solutions of $F$ is still the solution of Eqs.(A.6)-(A.7).

For the dark energy models, the equation of state parameter $w$ is a constant and $w<-\frac{1}{3}[15]$. The pressure $p$, the energy density $\rho$, and the state parameter $w$ are connected by

$$
w=\frac{p}{\rho} .
$$

We can express the energy-momentum tensor for dark energy as follows [29],

$$
\begin{aligned}
& T_{t}^{t}=\rho(r), \\
& T_{i}^{j}=3 w \rho(r)\left[-(1+3 \beta) \frac{r_{i} r^{j}}{r_{n} r^{n}}+\beta \delta_{i}{ }^{j}\right],
\end{aligned}
$$


where $\beta$ will be determined by the dark energy spacetime. We see that the space-component is proportional to the time-component. Averaging over the angles gives,

$$
\left\langle T_{i}{ }^{j}\right\rangle=-\rho(r) w \delta_{i}{ }^{j}=-p(r) \delta_{i}{ }^{j},
$$

where $\left\langle r_{i} r^{j}\right\rangle=\frac{1}{3} \delta_{i}{ }^{j} r_{n} r^{n}$ [29]. The above formulas could include matter contribution with $\rho_{\mathrm{M}}=M \delta^{3}(\vec{r})$, which has no contribution to $(p, w)$ for $r \neq 0$.

Using the condition of linearity and additivity can fix the parameter in Eq.(A.10), $\beta=-(3 w+1) /(6 w)$, which leads to [29]

$$
\begin{aligned}
T_{t}{ }^{t} & =T_{r}{ }^{r}=\rho, \\
T_{\theta}{ }^{\theta} & =T_{\phi}{ }^{\phi}=-\frac{\rho}{2}(3 w+1)=-\frac{1}{2}(3 p+\rho) .
\end{aligned}
$$

Combining Eqs.(A.6)-(A.7) with the expressions (A.12)-(A.13) leads to the equation,

$$
r^{2} F^{\prime \prime}+3(w+1) r F^{\prime}+(3 w+1) F=0
$$

which has the following solutions,

$$
F_{\mathrm{DE}}=-2\left(\frac{r_{o}}{r}\right)^{3 w+1}, \quad F_{\mathrm{N}}=-2 \frac{r_{g}}{r},
$$

where $r_{o}$ and $r_{g}$ are the integral constants serving as the normalization scale-factors. Thus, the sum of the above solutions $F=F_{\mathrm{N}}+F_{\mathrm{DE}} \equiv 2 \Phi$ is also the solution due to the linearity and additivity. Hence, we can express the metric parameters $\mathbb{A}=-\mathbb{B}=\ln (1+F)=\ln (1+2 \Phi)$ as follows,

$$
\begin{aligned}
e^{\mathbb{A}} & =e^{-\mathbb{B}}=1+2 \Phi \\
\Phi & =-\frac{r_{g}}{r}-\left(\frac{r_{o}}{r}\right)^{3 w+1} \equiv \Phi_{\mathrm{N}}+\Phi_{\mathrm{DE}},
\end{aligned}
$$

where in Eq.(A.17) the first term $\Phi_{\mathrm{N}}$ just corresponds to the Newtonian potential with mass $r_{g}=M$, and the constant $r_{o}$ in the $\Phi_{\mathrm{DE}}$ term characterizes the size of the present universe.

Substituting Eqs.(A.16)-(A.17) into Eq.(A.1), we derive the spacetime metric,

$$
\mathrm{d} S^{2}=(1+2 \Phi) \mathrm{d} t^{2}-\frac{\mathrm{d} r^{2}}{(1+2 \Phi)}-r^{2}\left(\mathrm{~d} \theta^{2}+\sin ^{2} \theta \mathrm{d} \phi^{2}\right),
$$

with the gravitational potential $\Phi=\Phi_{\mathrm{N}}+\Phi_{\mathrm{DE}}$,

$$
\Phi_{\mathrm{N}}=-\frac{M}{r}, \quad \Phi_{\mathrm{DE}}=-\left(\frac{r_{o}}{r}\right)^{3 w+1} .
$$

The above spacetime metric (A.18) reproduces Eq.(2.1) up to linear order of $\Phi$ under the post-Newtonian approximation, and the potential (A.19) coincides with Eq.(2.2). For the special case of cosmological constant $(w=-1)$ as dark energy, the metric (A.18) reduces to that of the familiar Schwarzschild-de Sitter (SdS) spacetime. For convenience, we will denote Eqs.(A.18)-(A.19) as SdSw metric. 
The general formulas (A.18)-(A.19) give the following Newtonian gravitational force and dark energy force,

$$
\vec{F}=-\nabla \Phi=\left(-\frac{M}{r^{2}}+\frac{|3 w+1|}{r_{o}}\left(\frac{r}{r_{o}}\right)^{|3 w+2|}\right) \hat{\boldsymbol{r}},
$$

where the current data [15] requires $3 w+1<0$ at $12.7 \sigma$ level and $3 w+2<0$ at $6.1 \sigma$ level. Hence, the dark energy force is always repulsive and increases with distance $r$. Requiring the cancellation between the Newtonian and dark energy forces $\vec{F}=0$, we derive the critical radius,

$$
r_{\mathrm{cri}}=r_{o}\left(\frac{M}{|3 w+1| r_{o}}\right)^{-\frac{1}{3 w}} .
$$

This reproduces Eq.(4.2) in Sec. 4. At the scale of $r \lesssim r_{\text {cri }}$, the matter contribution is larger than that of dark energy, and the matter distribution has significant structures. Thus, at such scales, the cosmological principle no longer holds and the FLRW metric is not a valid description of the spacetime. This means that for $r \lesssim r_{\text {cri }}$, it is not justified to use FLRW metric with matter density treated as small perturbation. As we demonstrated above, for the scale $r \lesssim r_{\text {cri }}$, the spacetime geometry is best described by the $\operatorname{SdS} w$ metric (A.18) [or (2.1)] with the gravitational potential (A.19) [or (2.2)]. On the other hand, with the key concept of critical radius (A.21), we should define the (large) cosmological scale as $r^{3} \gg r_{\text {cri }}^{3}$, at which the cosmological principle will be realized. With the astronomical data, we may estimate the maximal critical radius $r_{\mathrm{cri}}^{\max }$ for the galaxy cluster with the largest mass $M_{\max }$, which sets the lower limit on the cosmological scales.

For the matter part, we have the conventional Poisson equation,

$$
-\nabla^{2} \Phi_{\mathrm{M}}=4 \pi\left(\rho_{\mathrm{M}}+3 p_{\mathrm{M}}\right)
$$

where the pressure $p_{\mathrm{M}}$ is negligible compared to the energy density $\rho_{\mathrm{M}}$.

With Eqs.(A.12)-(A.13), the energy-momentum tensor of dark energy takes the following form,

$$
\left\langle T_{\mu}{ }^{\nu}\right\rangle=\operatorname{diag}\left(\rho_{w},-p_{w},-p_{w},-p_{w}\right),
$$

where $\rho_{w}=\rho_{\mathrm{DE}}$ is the dark energy density and $p_{w}=p_{\mathrm{DE}}$ denotes its pressure, with the state parameter $w$. From Eq.(A.6) and Eqs. (A.9),(A.19), we derive

$$
-\nabla^{2} \Phi_{\mathrm{DE}}=-4 \pi \rho_{w}
$$

Thus, we may combine Eqs.(A.22) and (A.24),

$$
-\nabla^{2} \Phi=4 \pi\left(\rho_{\mathrm{M}}-\rho_{w}\right),
$$

where we have dropped the pressure term $p_{\mathrm{M}}$ since $p_{\mathrm{M}} \ll \rho_{\mathrm{M}}$ for non-relativistic Newtonian source $M$. Eq.(A.25) is the modified Poisson equation, which includes the contributions 
from both Newtonian gravity and dark energy. For the case of cosmological constant as dark energy, we have $w=-1$ and $\rho_{w}=\frac{\Lambda}{4 \pi}$. Thus, in this special case, the Poisson equation (A.25) reduces to the form,

$$
-\nabla^{2} \Phi=4 \pi \rho_{\mathrm{M}}-\Lambda
$$

which agrees with [18].

In general, the potential $\Phi_{\mathrm{DE}}$ in Eq.(A.19) can describe different dark energy models characterized by the equation of state parameter: $-1<w<-\frac{1}{3}$ for the quintessence model, $w<-1$ for the phantom model, and $w=-1$ for the cosmological constant model.

\section{A.2 Phantom Model}

In the phantom model, the equation of state parameter $w_{p}$ is a constant with $w_{p}<-1$. The pressure $p_{p}$, the energy density $\rho_{p}$, and the state parameter $w_{p}$ are given by

$$
\begin{aligned}
\rho_{p} & =-\frac{\dot{\phi}^{2}}{2}+V(\phi), \\
p_{p} & =-\frac{\dot{\phi}^{2}}{2}-V(\phi), \\
w_{p} & =\frac{p_{p}}{\rho_{p}},
\end{aligned}
$$

where $V(\phi)$ is the potential of phantom field. From Eq.(A.19), we have the dark energy potential

$$
\Phi_{p}=-\left(\frac{r_{o}}{r}\right)^{3 w_{p}+1}
$$

\section{A.3 Quintessence Model}

In the quintessence model, the pressure $p_{q}$, the energy density $\rho_{q}$, and the state parameter $w_{q}$ are expressed as follows,

$$
\begin{aligned}
& \rho_{q}=\frac{\dot{\phi}^{2}}{2}+V(\phi), \\
& p_{q}=\frac{\dot{\phi}^{2}}{2}-V(\phi), \\
& w_{q}=\frac{p_{q}}{\rho_{q}},
\end{aligned}
$$

where $V(\phi)$ is the potential of quintessence field.

After exchanging $\rho_{q}$ with $-p_{p}$, and $p_{q}$ with $-\rho_{q}$, and following the above derivation, we have

$$
\begin{aligned}
\rho_{q} \leftrightarrow-p_{p}, \quad p_{q} \leftrightarrow-\rho_{p}, \\
w_{q}=\frac{p_{q}}{\rho_{q}}=\frac{-\rho_{p}}{-p_{p}}=\frac{1}{w_{p}} .
\end{aligned}
$$


For the quintessence spacetime, the state parameter $w_{q}$ is a constant within the range $-1<w_{q}<-\frac{1}{3}$.

Similar to the case of phantom model, we have the quintessence dark energy potential from Eq.(A.19),

$$
\Phi_{q}=-\left(\frac{r_{o}}{r}\right)^{3 w_{q}+1}
$$

\section{A.4 Cosmological Constant Model}

In the case of the cosmological constant model of dark energy $(\Lambda>0)$, the equation of state parameter is given by $w=-1$. Thus, from the general form in Eq.(A.19), we have the dark energy potential $\Phi_{\mathrm{DE}}$ reduced to

$$
\Phi_{\Lambda}=-\left(\frac{r}{r_{o}}\right)^{2}=-\frac{\Lambda}{6} r^{2},
$$

with the scale factor $r_{o}=\sqrt{6 / \Lambda}$, which is consistent with [17][18].

For the cosmological constant model of $\Lambda>0$, the generic spacetime metric (A.18) reduces to the SdS geometry, ${ }^{3}$

$$
\mathrm{d} S^{2}=\left(1-\frac{2 M}{r}-\frac{\Lambda r^{2}}{3}\right) \mathrm{d} t^{2}-\frac{\mathrm{d} r^{2}}{\left(1-\frac{2 M}{r}-\frac{\Lambda r^{2}}{3}\right)}-r^{2}\left(\mathrm{~d} \theta^{2}+\sin ^{2} \theta \mathrm{d} \phi^{2}\right) .
$$

In Sec. 4 , we explicitly derived in Eq.(4.9) the cosmological constant contribution $\Delta \alpha_{\mathrm{DE}}$ to the light deflection in the region $r \lesssim r_{\text {eff }} \sim r_{\text {cri }}$ of the isolated lensing system. In the following, we analyze the region beyond this lensing system with $r^{3} / r_{\text {cri }}^{3} \gg 1$, which corresponds to $\Phi_{\mathrm{N}} / \Phi_{\mathrm{DE}} \sim r_{\text {cri }}^{3} / r^{3} \ll 1$. For this region, the Newtonian potential term $\Phi_{\mathrm{N}}$ becomes negligible, and thus the SdS geometry (A.38) reduces to the de Sitter metric,

$$
\begin{aligned}
\mathrm{d} S^{2} & \simeq \mathrm{d} S_{\mathrm{dS}}^{2}=\left(1-\frac{\Lambda r^{2}}{3}\right) \mathrm{d} t^{2}-\frac{\mathrm{d} r^{2}}{\left(1-\frac{\Lambda r^{2}}{3}\right)}-r^{2}\left(\mathrm{~d} \theta^{2}+\sin ^{2} \theta \mathrm{d} \phi^{2}\right) \\
& =\mathrm{d} t^{\prime 2}-\frac{\mathrm{d} r^{2}}{\left(1-\frac{\Lambda r^{2}}{3}\right)}-r^{2}\left(\mathrm{~d} \theta^{2}+\sin ^{2} \theta \mathrm{d} \phi^{2}\right)
\end{aligned}
$$

where $\mathrm{d} t=\left(1-\frac{\Lambda r^{2}}{3}\right)^{-\frac{1}{2}} \mathrm{~d} t^{\prime}$. Let us make the conformal transformation, $r=\bar{r} /\left(1+\frac{\Lambda}{12} \bar{r}^{2}\right)=$ $\bar{r} /\left(1+\frac{1}{2} \frac{\bar{r}^{2}}{r_{o}^{2}}\right) \simeq \bar{r}$ and $\mathrm{d} t^{\prime}=\bar{R}(\bar{r}) \mathrm{d} \tau$ with $\bar{R}(\bar{r}) \equiv 1 /\left(1+\frac{1}{2} \frac{\bar{r}^{2}}{r_{o}^{2}}\right) \simeq 1$, where $\tau$ is the conformal time, and the approximation sign $\simeq$ is due to $r^{2}, \bar{r}^{2} \ll r_{o}^{2}$ for any typical cosmological scale $r$ or $\bar{r}$. With this transformation, we have

$$
\begin{aligned}
\mathrm{d} S_{\mathrm{dS}}^{2} & =\bar{R}^{2}(\bar{r})\left[\mathrm{d} \tau^{2}-\mathrm{d} \bar{r}^{2}-\bar{r}^{2}\left(\mathrm{~d} \theta^{2}+\sin ^{2} \theta \mathrm{d} \phi^{2}\right)\right]=\bar{R}^{2} \mathrm{~d} S_{\text {Mink }}^{2} \\
& \simeq \mathrm{d} S_{\text {Mink }}^{2},
\end{aligned}
$$

\footnotetext{
${ }^{3}$ Eq.(A.38) is called the Schwarzschild-anti-de Sitter metric for $\Lambda<0$, and is known as Kottler metric [30] for a generic cosmological constant $\Lambda$ which can be either positive or negative.
} 
where $\mathrm{d} S_{\text {Mink }}^{2}$ is the usual Minkowski spacetime. In the above, the de Sitter metric $g_{\mu \nu}$ is connected to the Minkowski metric $\eta_{\mu \nu}$ via conformal transformation $g_{\mu \nu}=\bar{R}^{2} \eta_{\mu \nu}$. In the second line of Eq.(A.40), we have used the fact that the conformal transformation factor $\bar{R} \simeq 1$ due to $r^{2} \ll r_{o}^{2}$. From these, we see that the de Sitter spacetime is conformally flat, and also has approximate flatness under $r^{2} \ll r_{o}^{2}$. Combining Eqs.(A.39) and (A.40), we have proven that the spacetime outside the lensing region, obeying both $r^{3} / r_{\text {cri }}^{3} \gg 1$ and $r^{2} / r_{o}^{2} \ll 1$, is approximately flat.

On the other hand, the flat FLRW spacetime corresponds to the FLRW metric with spatial curvature $K=0$,

$$
\mathrm{d} \bar{S}_{\mathrm{FLRW}}^{2}=a^{2}(\tau)\left[\mathrm{d} \tau^{2}-\mathrm{d} \bar{r}^{2}-\bar{r}^{2}\left(\mathrm{~d} \theta^{2}+\sin ^{2} \theta \mathrm{d} \phi^{2}\right)\right]
$$

Comparing Eqs. (A.40) with (A.41), we see that the de Sitter spacetime is conformally equivalent to a flat FLRW metric with vanishing spatial curvature $(K=0),{ }^{4}$

$$
\mathrm{d} \bar{S}_{\mathrm{FLRW}}^{2}=[a(\tau) / \bar{R}(\bar{r})]^{2} \mathrm{~d} S_{\mathrm{dS}}^{2} \simeq a^{2}(\tau) \mathrm{d} S_{\mathrm{dS}}^{2} .
$$

This shows that the deSitter spacetime can be conformally and isometrically embedded into the flat FLRW spacetime. Hence, for $r^{3} / r_{\text {cri }}^{3} \gg 1$, the SdS metric is conformally equivalent to a flat FLRW metric which has no effect on the deflection because of its flatness (Bartelmann and Schneider 2001[4]). (We will further extend the above analysis to the case of $w \neq-1$ in Appendix C.2.) From these, the deflection region, $r \lesssim r_{\text {eff }}=O\left(r_{\text {cri }}\right)$, is well isolated from the flat FLRW spacetime, where $r_{\text {cri }} \simeq \bar{r}_{\text {cri }}$. For the transition region with $r \sim r_{\text {cri }}$, Eqs.(2.1)-(2.2) and (A.37) approximately hold. So, the metric outside the lensing region is fairly conformally flat, and has negligible effect on the light deflection. For an isolated lensing system, the lensing region of $r \lesssim r_{\text {cri }}$ is well approximated by Eq.(2.1) where the cosmological expansion effect becomes negligible so that $a(\tau) \simeq$ constant. In fact, for the analyses at astrophysical scales (such as galaxies or galaxy clusters), it is well justified to treat $a(\tau) \simeq$ constant for the data fitting [22][23], as we will further explain in Appendix C.1. Comparing Eq.(A.39) with Eq.(A.42), we see that at large scales with $r^{3} \gg r_{\text {cri }}^{3}$, the original SdS metric (A.38) becomes approximately equivalent to the FLRW metric (A.41) with $K=0$. Hence, the deflection angle (4.8)-(4.9) (generated in the lensing system $\left.r \lesssim r_{\text {eff }} \sim r_{\text {cri }}\right)$ will not be affected when the light ray travels to the faraway region $r^{3} \gg r_{\text {cri }}^{3}$. This is expected because the regions with approximately flat spacetime do not cause visible light deflection. Also, any conformal transformation [such as (A.42) or (A.40)] does retain the angles unchanged.

We note that the conventional lensing analyses [4] choose the FLRW metric with matter density treated as small perturbation. The observational data show that the FLRW metric is nearly flat $(K \simeq 0)$, while the matter perturbation only generates the Newtonian deflection.

\footnotetext{
${ }^{4}$ The equivalence between the deSitter metric (A.39) and the FLRW metric (A.41) (with $K=0$ ) under conformal transformation was known before and discussed in Ref. [31].
} 
So, for the region with $r^{3} / r_{\text {cri }}^{3} \gg 1$, it is clear that the light bending effect of the cosmological constant $\Lambda$ is either absent or negligibly small due to the flatness of the FLRW metric, as often found in the literature [8]. On the other hand, people usually restrict the deflection region to be nearby the immediate neighborhood of the matter distribution (with $r \ll r_{\text {cri }}$ ), rather than the effective region $0.3 r_{\text {cri }}<r<r_{\text {eff }} \sim r_{\text {cri }}$ (as we studied in Sec. 4 and Fig. 4). This is another reason that some literature [8] found the $\Lambda$ bending effect to be vanishing or negligible nearby the matter distribution. The similar point was also clarified before by Ishak, Rindler and Dossett [17].

Before concluding this Appendix, we note that the lensing effect of $\Lambda$ on light bending was considered before via different approaches as reviewed by Ishak and Rindler [8], who clarified some debates in the literature [9][17][28][32-35]. A main cause of the debates is the lack of a consistent approach which could treat the lensing system as a well-defined isolated system characterized by its critical radius $r_{\text {cri }}$ (rather than by the radius of the matter distribution of the lens). It was not well realized that within this isolated system, the matter potential and dark energy potential should be treated on equal footing and solved together from the Einstein equation under SdS metric (rather than the nearly flat FLRW metric of large cosmological scales); while for regions outside this system with $r^{3} / r_{\text {cri }}^{3} \gg 1$, the spacetime reduces to the deSitter metric and conformally recovers the FLRW metric (with $K=0$ ). Our approach provides an independent and conceptually clean resolution to this issue, which also favors Ishak and Rindler [8].

In the following, we give some further clarifications on the literature, making it clear that these do not affect our independent and self-contained approach. (i). Islam [32] noticed that $\Lambda$ does not modify the light orbital equation (5.4) (a second order ODE) and thought that $\Lambda$ does not affect light bending. Then, Rindler and Ishak [9] first found that $\Lambda$ can still generate light bending via the metric itself although Eq.(5.4) does not depend on $\Lambda$. Ref. [28] further noted that the light orbital equation in its form of the first-order ODE (5.5) does contain an explicit $\Lambda$ term, but it could be absorbed into the definition of an effective impact parameter $B$ so that (5.5) takes the form (5.6). This seems to make the $\Lambda$ effect not directly testable. They also made the thin lens approximation to absorb the $\Lambda$ effect into angular diameter distance via $B \approx D_{C \mathcal{O}} \theta$. But this thin lens relation does not generally hold when $\theta_{c}$ is significant (cf. our Fig. 2). We point out that $B$ could make a sense as an effective impact parameter only for the special case of $w=-1$ and point-like light source. For all dark energy models with $w \neq-1$, the $w$-dependent term in Eq.(5.1) or (5.2) is $r$-dependent and a universal effective impact parameter $B$ does not exist. As we clarified below Eq.(5.6), it holds only for point-like light source, but not the more realistic $2 \mathrm{~d}$ sources. The impact parameter $b$ of such $2 \mathrm{~d}$ sources varies its value on the surface of the source. Hence, no universal effective impact parameter $B$ exists to fully absorb $\Lambda$ effect, and fitting the lensing data from 2 d light sources can discriminate the $\Lambda$-induced deflection effect. (ii). Ishak [33] also analyzed the $\Lambda$ contribution to 
light bending and time delays from integrating the potential term as well as from the Fermat's principle. The pure de Sitter metric was used for calculations inside the vacuole (the effective lensing area). This appears not well justified since the pure de Sitter metric is conformally flat [cf. our Eq.(A.40)], and thus does not cause light bending. (iii). Under the weak deflection approximation, Refs. [8] [9] found the $\Lambda$-induced light bending, $\Delta \alpha_{\Lambda}=-\frac{\Lambda R^{3}}{12 M}$, which diverges as $M \rightarrow 0$ and appears inconsistent with the weak deflection assumption. As we clarified at the end of Sec. 3 and in the paragraph below Eq.(4.9), the deflection $\Delta \alpha_{\mathrm{DE}}$ consistently vanishes in the limit $M \rightarrow 0$, as expected. (iv). In the lensing system of $r \lesssim r_{\text {cri }}$, the matter contribution is larger than that of dark energy, and the matter distribution has significant structures. Hence, the cosmological principle no longer holds at such scales and here the FLRW metric is not a valid description of the spacetime. This means that for $r \lesssim r_{\text {cri }}$, it is not justified to use FLRW metric with matter density treated as small perturbation. As shown in Appendices A.1 and A.4, the spacetime geometry in the region $r \lesssim r_{\text {cri }}$ is best described by the metric (A.18) [or (2.1)] with the gravitational potential (A.19) [or (2.2)], which reduces to the SdS metric (A.38) with dark energy potential (A.37) for the cosmological constant case $(w=-1)$. (v). Simpson et al. in [35] defined the vacuole boundary of lensing system by requiring the potential $\Phi=0$ at $r=R$ below their Eq.(15). Then, they derived light bending angle $\alpha=\alpha_{\mathrm{M}}+\alpha_{\Lambda}=\left(4 M / R-\Lambda R r_{b} / 3\right) \sqrt{1-R^{2} / r_{b}^{2}}$ in their Eq.(32), where both $\alpha_{\mathrm{M}}=0$ and $\alpha_{\Lambda}=0$ hold at the boundary $R=r_{b}$. This appears inconsistent because it even makes the conventional matter-induced light bending $\alpha_{\mathrm{M}}$ (à la Einstein [10]) vanish. Moreover, imposing the condition $\Phi=0$ at the vacuole boundary appears improper because our gravitational potential (2.2) (derived from the Einstein equation in Appendix A.1) proves that both the Newtonian and dark energy terms always share the same sign and cannot cancel each other to give $\Phi=0$. In fact, as we showed in Eq.(A.20) and Eq.(4.2) (including the special case $w=-1$ ), the Newtonian force and the dark energy force (rather than their potential terms) exactly cancel at the critical radius $r_{\text {cri }}$. Hence, the boundary of an isolated lensing system should be correctly characterized by the effective radius around its critical radius, $r_{\text {eff }} \sim r_{\text {cri }}$. (vi). In the literature [35], it is sometimes argued that the cosmological comoving observer has a local Hubble velocity $v_{L} \simeq H D_{L}$ (with $D_{L}$ the luminosity distance from the observor), which causes an aberration factor to the deflection. In fact, the Hubble velocity is not a real physical velocity, and does not apply to the aberration equation. It is just an apparent velocity and is solely determined by the expansion factor $a(t)$, so it is a cosmological expansion effect. The factor $a(t)$ causes no visible effect on the deflection, especially the ratio $\Delta \alpha_{\mathrm{DE}} / \alpha_{\mathrm{M}}$ [17], because the expansion is conformally flat for $K \simeq 0$, as discussed around Eqs.(A.39)-(A.42). 


\section{B Deriving Light Orbital Equation for Generic State Parameter w}

In this Appendix, we will derive the new light orbital equation (5.1) for generic state parameter $w$ and under the $\mathrm{SdS} w$ metric.

For a gravitational lensing system with generical dark energy state parameter $w$, we have the $\mathrm{SdS} w$ spacetime metric from Eq.(A.18),

$$
\mathrm{d} S^{2}=\left[1-2 \frac{M}{r}-2\left(\frac{r_{o}}{r}\right)^{3 w+1}\right] \mathrm{d} t^{2}-\frac{\mathrm{d} r^{2}}{\left[1-2 \frac{M}{r}-2\left(\frac{r_{o}}{r}\right)^{3 w+1}\right]}-r^{2}\left(\mathrm{~d} \theta^{2}+\sin ^{2} \theta \mathrm{d} \phi^{2}\right) .
$$

Let us define the energy-momentum four-vector $K^{\mu}=\mathrm{d} x^{\mu} / \mathrm{d} \lambda$, where the $\lambda$ is an affine parameter. From Eq.(B.1), we have

$$
g_{00}=1-2 \frac{M}{r}-2\left(\frac{r_{o}}{r}\right)^{3 w+1}, \quad g_{11}=-g_{00}^{-1}, \quad g_{22}=-r^{2}, \quad g_{33}=-r^{2} \sin ^{2} \theta,
$$

and $g_{i j}=0$ for $i \neq j$. Since this metric respects the symmetries of time translation and space rotation, it holds the conservations of energy $E$ and angular momentum $L$, i.e., $E=$ constant and $L=$ constant. So we have,

$$
\begin{aligned}
& E=g_{0 \mu} K^{\mu}=\left[1-2 \frac{M}{r}-2\left(\frac{r_{o}}{r}\right)^{3 w+1}\right] \frac{\mathrm{d} t}{\mathrm{~d} \lambda}, \\
& L=g_{3 \mu} K^{\mu}=-r^{2} \sin ^{2} \theta \frac{\mathrm{d} \phi}{\mathrm{d} \lambda} .
\end{aligned}
$$

Using the null condition $\mathrm{d} S^{2}=0$ for a light ray, we have

$$
\left[1-2 \frac{M}{r}-2\left(\frac{r_{o}}{r}\right)^{3 w+1}\right] \mathrm{d} t^{2}-\frac{\mathrm{d} r^{2}}{\left[1-2 \frac{M}{r}-2\left(\frac{r_{o}}{r}\right)^{3 w+1}\right]}-r^{2}\left(\mathrm{~d} \theta^{2}+\sin ^{2} \theta \mathrm{d} \phi^{2}\right)=0 .
$$

Without losing generality, we confine the motion in the plane of $\theta=\frac{\pi}{2}$. Thus, we can rewrite Eqs.(B.3) as follows,

$$
\begin{aligned}
\frac{\mathrm{d} t}{\mathrm{~d} \lambda} & =E\left[1-2 \frac{M}{r}-2\left(\frac{r_{o}}{r}\right)^{3 w+1}\right]^{-1}, \\
\frac{\mathrm{d} \phi}{\mathrm{d} \lambda} & =-\frac{L}{r^{2}} .
\end{aligned}
$$

With these, we further rederive Eq.(B.4) as

$$
\left(\frac{\mathrm{d} r}{\mathrm{~d} \lambda}\right)^{2}=E^{2}-\frac{L^{2}}{r^{2}}\left[1-2 \frac{M}{r}-2\left(\frac{r_{o}}{r}\right)^{3 w+1}\right] .
$$

Substituting the second formula of Eq.(B.5) into Eq.(B.6), we deduce the following light orbital equation for general state parameter $w$,

$$
\left(\frac{1}{r^{2}} \frac{\mathrm{d} r}{\mathrm{~d} \phi}\right)^{2}=\frac{E^{2}}{L^{2}}-\frac{1}{r^{2}}\left[1-2 \frac{M}{r}-2\left(\frac{r_{o}}{r}\right)^{3 w+1}\right]
$$

For convenience, let us define the notations $u=1 / r$ and $b=L / E$. Hence, we can reexpress Eq.(B.7) as follows,

$$
\left(\frac{\mathrm{d} u}{\mathrm{~d} \phi}\right)^{2}=\frac{1}{b^{2}}-u^{2}+2 M u^{3}+2 r_{o}^{3 w+1} u^{3(w+1)},
$$

which just reproduces the generical light orbital equation (5.1) which we presented in Sec. 5. 


\section{Analyses within and outside the Lensing System}

In this Appendix, we first show that the cosmic expansion effect is negligible for a lensing system at typical astrophysical scales. Then, we will prove the approximate conformal flatness of the $\mathrm{SdS} w$ metric in the region $r^{3} / r_{\mathrm{cri}}^{3} \gg 1$.

\section{C.1 Cosmic Expansion Factor is Nearly Constant at Astrophysical Scales}

In the following, we show that for a lensing system at typical astrophysical scales (such as galaxies or galaxy clusters), the cosmic expansion factor is nearly constant. Although this is a known fact, it useful to explicitly clarify it here for supporting the current formulation.

For a given astrophysical object, its luminosity distance from us as the observer is denoted as $D_{L}$, and its size is denoted as $\Delta D_{L}$. According to the Hubble Law, we have

$$
z \simeq v_{L}=H D_{L}
$$

where the $v_{L}$ is the velocity along the sight direction, the $z$ is the cosmological redshift. Then, we also have

$$
\Delta z \simeq H \Delta D_{L}
$$

where the $\Delta z$ describes the redshift fluctuation due to the finite size of this astrophysical object. Combining Eqs.(C.1) and (C.2), we deduce

$$
\frac{\Delta z}{z} \simeq \frac{\Delta D_{L}}{D_{L}}
$$

Let $a=a(\tau)$ be the expansion factor at redshift $z$, and $a_{0}=a\left(\tau_{0}\right)$ be the expansion factor at $z=0$. Thus, we have

$$
\frac{a}{a_{0}}=\frac{1}{1+z} .
$$

The redshift fluctuation $\Delta z$ in Eq.(C.2) corresponds to a variation $\Delta a$ of the cosmic expansion factor $a$. Including this effect, we reexpress the formula (C.4),

$$
\frac{a+\Delta a}{a_{0}}=\frac{1}{1+z+\Delta z} .
$$

With Eqs. (C.4) and (C.5), we deduce

$$
\frac{|\Delta a|}{a}=\frac{|\Delta z|}{1+z} \simeq \frac{\left|\Delta D_{L}\right|}{D_{L}} \frac{z}{1+z} .
$$

The galaxies and galaxy clusters are the gravitationally self-bounded systems in the Universe, with typical mass range $M=\left(10^{12}-10^{16}\right) M_{\odot}$. In Sec. 4 , we estimated the range of their critical radii, $r_{\text {cri }} \simeq(1.1-23) \mathrm{Mpc}$, for $w=-1$. Thus, we can expect $\Delta D_{L} \lesssim r_{\text {cri }} \simeq$ $(1.1-23)$ Mpc. For instance, we consider the current lensing experiments for galaxies with masses $M \lesssim 10^{13} M_{\odot}$ and at redshift $z=0.1-0.5[22,23]$. We can estimate $D_{L}=z / H \simeq$ 
$(0.42-2.1) \times 10^{3} \mathrm{Mpc}$ and $\Delta D_{L} \lesssim r_{\text {cri }} \lesssim 2.3 \mathrm{Mpc}$. Thus, we have $\frac{|\Delta z|}{z} \lesssim(5.5-1.1) \times 10^{-3}$ and $\frac{|\Delta a|}{a} \lesssim(5.0-3.7) \times 10^{-4} \ll 1$. Then, we consider the galaxies or galaxy clusters with masses $M=10^{12-16} M_{\odot}$ and at redshift $z=0.5-2[24-26]$. Thus, $D_{L}=z / H \simeq(2.1-8.4) \times 10^{3} \mathrm{Mpc}$ for $z=0.5-2$, and $\Delta D_{L} \lesssim r_{\text {cri }} \simeq(1.1-23)$ Mpc for $M=10^{12-16} M_{\odot}$. Thus, we can estimate $\frac{|\Delta z|}{z} \lesssim(0.52-11) \times 10^{-3}$ and $\frac{|\Delta a|}{a} \lesssim(0.17-3.7) \times 10^{-3} \ll 1$ at $z=0.5$; while $\frac{|\Delta z|}{z} \lesssim(0.13-2.7) \times 10^{-3}$ and $\frac{|\Delta a|}{a} \lesssim(0.08-1.8) \times 10^{-3} \ll 1$ at $z=2$. The above analysis shows that for a typical galaxy or galaxy cluster as the gravitational lensing, the cosmic expansion effect is negligible and the lensing system is well described by the static $\mathrm{SdS} w$ spacetime (A.18)-(A.19).

\section{C.2 Approximate Conformal Flatness for Generic State Parameter w}

In this Appendix, we show that for the region outside the lensing system with $r^{3} / r_{\text {cri }}^{3} \gg 1$, the general $\mathrm{SdS} w$ metric (A.18)-(A.19) has approximate conformal flatness. This is an extension of our discussion in Appendix A.4. Since $w$ is quite close to $w=-1$ [15], we see that the condition $r^{3} / r_{\text {cri }}^{3} \gg 1$ lead to $\Phi_{\mathrm{N}} / \Phi_{\mathrm{DE}} \sim r_{\text {cri }}^{3|w|} / r^{3|w|} \ll 1$. Hence, the Newtonian potential $\Phi_{\mathrm{N}}$ becomes negligible for $r^{3} / r_{\text {cri }}^{3} \gg 1$, and thus the $\operatorname{SdS} w$ metric (A.18)-(A.19) approximately reduces to the de Sitter spacetime with generical $w$ (denoted as $\mathrm{dS} w$ ),

$$
\begin{aligned}
\mathrm{d} S^{2} \simeq \mathrm{d} S_{\mathrm{d} S w}^{2} & =\left[1-2\left(\frac{r_{o}}{r}\right)^{3 w+1}\right] \mathrm{d} t^{2}-\frac{\mathrm{d} r^{2}}{\left[1-2\left(\frac{r_{o}}{r}\right)^{3 w+1}\right]}-r^{2}\left(\mathrm{~d} \theta^{2}+\sin ^{2} \theta \mathrm{d} \phi^{2}\right) \\
& =\mathrm{d} t^{\prime 2}-\frac{\mathrm{d} r^{2}}{\left[1-2\left(\frac{r_{o}}{r}\right)^{3 w+1}\right]}-r^{2}\left(\mathrm{~d} \theta^{2}+\sin ^{2} \theta \mathrm{d} \phi^{2}\right),
\end{aligned}
$$

where $\mathrm{d} t=\left[1-2\left(\frac{r_{o}}{r}\right)^{3 w+1}\right]^{-\frac{1}{2}} \mathrm{~d} t^{\prime}$. Then, we make the coordinate transformations, $r=\bar{R}(\bar{r}) \bar{r}$ and $\mathrm{d} t^{\prime}=\bar{R}(\bar{r}) \mathrm{d} \tau$, where $\tau$ is the conformal time and $\bar{R}(\bar{r})$ obeys the condition,

$$
\frac{\mathrm{d} \ln (\hat{r} \bar{R})}{\mathrm{d} \ln \hat{r}}=\left[1-2(\hat{r} \bar{R})^{-(3 w+1)}\right]^{\frac{1}{2}}
$$

where $\hat{r} \equiv \bar{r} / r_{o}$. From Eq.(C.8), we derive the solution

$$
\begin{aligned}
\bar{R}(\bar{r}) & =\frac{r_{o}}{\bar{r}}\left[\frac{1}{2} \sin ^{2}\left(2 \arctan \frac{\left(\bar{r} / r_{o}\right)^{\frac{|3 w+1|}{2}}}{\sqrt{2}}\right)\right]^{\frac{1}{|3 w+1|}} \\
& \simeq 1-\frac{\left(\bar{r} / r_{o}\right)^{|3 w+1|}}{|3 w+1|} \simeq 1, \quad\left(\text { for } \bar{r}^{2} \ll r_{o}^{2}\right),
\end{aligned}
$$

where $3 w+1<0$ always holds due to the current data [15]. For the special case of $w=-1$, the formula (C.9) reduces to $\bar{R}(\bar{r})=1 /\left[1+\frac{1}{2}\left(\bar{r}^{2} / r_{o}^{2}\right)\right]$, which agrees to what shown below Eq.(A.39).

Under the above coordinate transformations $\left(t^{\prime}, r\right) \rightarrow(\tau, \bar{r})$, we can reexpress the metric (C.7) as follows

$$
\mathrm{d} S_{\mathrm{dS} w}^{2}=\bar{R}^{2}(\bar{r})\left[\mathrm{d} \tau^{2}-\mathrm{d} \bar{r}^{2}-\bar{r}^{2}\left(\mathrm{~d} \theta^{2}+\sin ^{2} \theta \mathrm{d} \phi^{2}\right)\right]=\bar{R}^{2} \mathrm{~d} S_{\text {Mink }}^{2}
$$


which means

$$
\mathrm{d} \bar{S}_{\mathrm{FLRW}}^{2}=[a(\tau) / \bar{R}(\bar{r})]^{2} \mathrm{~d} S_{\mathrm{dS} w}^{2} .
$$

This shows that the $\mathrm{dS} w$ metric is equivalent to the flat FLRW metric $(K=0)$ under conformal transformation. Hence, from these we see that in the region $r^{3} / r_{\text {cri }}^{3} \gg 1$, the $\mathrm{SdS} w$ metric becomes conformally equivalent to a flat FLRW metric $(K=0)$, and thus causes no visible light deflection. The above is an extension of our discussion around Eqs.(A.38)-(A.42) of Appendix A.4 to the case with a generic state parameter $w$.

\section{References}

[1] P. A. R. Ade et al., [Planck Collaboration] Astron. Astrophys. 594 (2016) A13 [arXiv:1502.01589 [astro-ph.CO]].

[2] For reviews, e.g., D. H. Weinberg, M. J. Mortonson, D. J. Eisenstein, C. Hirata, A. G. Riess, E. Rozo, Phys. Rept. 530 (2013) 87 [arXiv:1201.2434 [astro-ph.CO]]; D. Weinberg, D. Bard, K. Dawson, O. Dore, J. Frieman, K. Gebhardt, M. Levi, J. Rhodes, Working Group Report of "Dark Energy and CMB", arXiv:1309.5380 [astro-ph.CO]; and references therein.

[3] For reviews, e.g., P. J.E. Peebles and B. Ratra, Rev. Mod. Phys. 75 (2003) 559 [astro-ph/0207347]; and references therein.

[4] For reviews, e.g., M. Bartelmann and P. Schneider, Phys. Rept. 340 (2001) 291 [arXiv:astro-ph /9912508 [astro-ph.CO]]; M. A. Troxel and M. Ishak, Phys. Rept. 558 (2014) 1 [arXiv:1407.6990 [astro-ph.CO]]; V. Perlick, Living Rev. Rel. 7 (2004) 9; and references therein.

[5] A. Heavens, Nucl. Phys. Proc. Suppl. 194 (2009) 76.

[6] For a review, e.g., M. Kilbinger, Rept. Prog. Phys. 78 (2015) 086901 [arXiv:1411.0115 [astro-ph.CO]]; and references therein.

[7] D. Walsh, R. F. Carswell, and R. J. Weymann,[24-26] Nature 279 (1979) 381.

[8] For a review, Mustapha Ishak and Wolfgang Rindler, Gen. Rel. Grav. 42 (2010) 2247 [arXiv:1006.0014 [astro-ph.CO]]; and references therein.

[9] Wolfgang Rindler and Mustapha Ishak, Phys. Rev. D 76 (2007) 043006 [arXiv:0709.2948 [astro-ph]].

[10] S. Weinberg, "Gravitation and Cosmology: Principles and Application of the General Theory of Relativity", by John Wiley \& Sons, New York, USA, 1972.

[11] For reviews, e.g., M. Li, X. D. Li, S. Wang and Y. Wang, Comm. Theor. Phys. 56 (2011) 3 [arXiv:1103.5870]; Y. F. Cai, E. N. Saridakis, M. R. Setare, and J. Q. Xia, Phys. Rept. 493 (2010) 1 [arXiv:0909.2776]; S. Wang, Y. Wang and M. Li, arXiv:1612.00345; and references therein.

[12] E.g., D. Lebedev and K. Lake, arXiv:1308.4931 [gr-qc]; and references therein.

[13] E.g., R. Blandford and R. Narayan, Astrophys. J. 310 (1986) 568;

P. Schneider, Astronomy \& Astrophysics 143 (1985) 413.

[14] J. Binney and M. Merrifield, "Galactic Astronomy", Princeton University Press, 1998, cf. chapter 2.4.

[15] E. Aubourg et al., Phys. Rev. D 92 (2015) 123516 [arXiv: 1411.1074 [astro-ph.CO]].

[16] M. Ishak, W. Rindler, J. Dossett, J. Moldenhauer, and C. Allison, Mon. Not. Roy. Astron. Soc. 388 (2008) 1279 [arXiv:0710.4726 [astro-ph]].

[17] Mustapha Ishak, Wolfgang Rindler, and Jason Dossett, Mon. Not. Roy. Astron. Soc. 403 (2010) 2152 [arXiv:0810.4956 [astro-ph]]. 
[18] C. M. Ho and S. D. H. Hsu, Astropart. Phys. 47 (2016) 74 [arXiv:1501.05952 [astro-ph.CO]].

[19] G. Darmois, Memorial de Sciences Mathematiques, Fascicule XXV, "Les equations de la gravitation einsteinienne", Chapitre V, (1927); W. Israel, Nuovo Cim B 44 (1966) 1; B 48 (1967) $463(\mathrm{E})$.

[20] A. Einstein and E. G. Straus, Rev. Mod. Phys. 17 (1945) 120; and 18 (1946) 148;

R. Balbinot, R. Bergamini, and A. Comastri, Phys. Rev. D 38 (1988) 2415.

[21] A. Einstein, "The Principle of Relativity", Annalen der Phys. 49 (1916) 769.

[22] M. Velander, E. van Uitert, H. Hoekstra et al., Mon. Not. Roy. Astron. Soc. 437 (2014) 2111 [arXiv:1304.4265 [astro-ph.CO]].

[23] Y. Shu, A. S. Bolton, S. Mao et al., Astrophysical Journal 833 (2016) 15 [arXiv:1608.08707 [astro-ph.GA]].

[24] A. Zitrin, T. Broadhurst, R. Barkana, Y. Rephaeli, and N. Benitez, Mon. Not. Roy. Astron. Soc. 410 (2011) 1939 [arXiv:1002.0521 [astro-ph.CO]].

[25] D. Sluse, V. Chantry, and P. Magain et. al., Astron. Astrophys. 538 (2012) A99 [arXiv:1112.0005 [astro-ph.CO]].

[26] K. Umetsu, A. Zitrin, D. Gruen, J. Merten, M. Donahue, and M. Postman, Astrophysical Journal 821 (2016) 116 [arXiv:1507.04385 [astro-ph.CO]].

[27] P. Bhattacharjee, S. Chaudhury, and S. Kundu, Astrophysical Journal 785 (2014) 1 [arXiv: 1310.2659 [astro-ph.GA]].

[28] H. Arakida and M. Kasai, Phys. Rev. D 85 (2012) 023006 [arXiv:1110.6735].

[29] V. V. Kiselev, Class. Quant. Grav. 20 (2003) 1187 [arXiv:gr-qc/0210040].

[30] F. Kottler, Ann. Phys. 361 (1918) 401.

[31] S. Weinberg, Cosmology, Oxford University Press (2008), see pp.44-45.

[32] N. J. Islam, Phys. Lett. A 97 (1983) 239.

[33] M. Ishak, Phys. Rev. D 78 (2008) 103006 [arXiv:0801.3514 [astro-ph]].

[34] M. Sereno, Phys. Rev. Lett. 102 (2009) 021301 [arXiv:0807.5123 [astro-ph]]; Phys. Rev. D 77 (2008) 043004 [arXiv:0711.1802 [astro-ph]];

[35] E.g., T. Schucker, Gen. Rel. Grav. 41 (2009) 67 [arXiv:0712.1559 [astro-ph]]; Gen. Rel. Grav. 41 (2009) 1595 [arXiv:0807.0380 [astro-ph]]; T. Schucker and N. Zaimen, Astron. Astrophys. 484 (2008) 103 [arXiv:0801.3776 [astro-ph]]; K. Lake, arXiv:0711.0673 [gr-qc]; F. Simpson, J. A. Peacock, A. F. Heavens, Mon. Not. Roy. Astron. Soc. 402 (2010) 2009 [arXiv:0809.1819 [astro-ph]]; A. Bhadra, S. Biswas, K. Sarkar, Phys. Rev. D 82 (2010) 063003 [arXiv:1007.3715 [gr-qc]]; H. Miraghaei and M. Nouri-Zonoz, Gen. Rel. Grav. 42 (2010) 2947 [arXiv:0810.2006 [gr-qc]]; T. Biressa and J. A. de Freitas Pacheco, Gen. Rel. Grav. 43 (2011) 2649 [arXiv:1105.3907 [gr-qc]]; F. Hammad, Mod. Phys. Lett. A 28 (2013) 1350181 [arXiv:1309.0263]; D. Lebedev and K. Lake, arXiv:1308.4931 [gr-qc]; D. Batic, S. Nelson, and M. Nowakowski, Phys. Rev. D 91 (2015) 104015 [arXiv:1412.3858 [gr-qc]]; M. Heydari-Fard, S. Mojahed, S. Y. Rokni, Astrophys. Space Sci. 351 (2014) 251; M. E. Aghili, B. Bolen, and L. Bombelli, Gen. Rel. Grav. 49 (2017) 10 [arXiv:1408.0786]; L. M. Butcher, Phys. Rev. D 94 (2016) 083011 [arXiv:1602.02751 [gr-qc]]; V. Faraoni and M. Lapierre-Leonard, Phys. Rev. D 95 (2017) 023509 [arXiv:1608.03164 [gr-qc]]; and references therein. 